\title{
Organisationsstruktur und Führungsstil nach Akquisitionen - eine empirische Untersuchung
}

\author{
Tobias Kretschmer $\mathbb{D} \cdot$ Christoph Dehne
}

Eingegangen: 2. September 2020 / Angenommen: 18. November 2020 / Online publiziert: 1. Dezember 2020

(C) Der/die Autor(en) 2020

Zusammenfassung Viele Akquisitionen bleiben hinter den in sie gesetzten Erwartungen zurück. Ein Grund hierfür kann sein, dass Synergien nur dann realisiert werden, wenn der Akquisition ein erfolgreicher Post-Merger-Integrationsprozess folgt, der häufig Veränderungen in der Organisationsstruktur und im Führungsstil impliziert. Anhand einer Stichprobe von 230 europäischen Unternehmen untersuchen wir die Tendenz der akquirierten Unternehmen, die Organisationsstruktur und den Führungsstil des akquirierenden Unternehmens zu übernehmen. Unsere Ergebnisse zeigen, dass Veränderungen hauptsächlich im akquirierten Unternehmen stattfinden und dass Veränderungen im Führungsstil oft durch Änderungen der Unternehmensstruktur bedingt werden.

Schlüsselwörter Organisationasstruktur · Führungstil · Akquisitionen · Produzierende Unternehmen · Integrationsprozesse nach Fusionen

\footnotetext{
T. Kretschmer $(\bowtie) \cdot$ C. Dehne

Fakultät für Betriebswirtschaftslehre, Institut für Strategie, Technologie und Organisation (ISTO),

Ludwig-Maximilians-Universität München (LMU), 80539 München, Deutschland

E-Mail: t.kretschmer@1mu.de

C. Dehne

E-Mail: christoph@dehne.org
} 


\title{
Organizational Structure and Leadership Style after Acquisitions - an Empirical Study
}

\begin{abstract}
Many acquisitions fail to reach their intended goal. One reason may be that synergies will only materialize if the acquisition is followed by a successful postmerger integration process that often implies a change in organizational structure and leadership style. Using a sample of 230 European firms, we study the proclivity of acquired firms to adopt the organizational structure and leadership style of the acquirer. Our results show that changes indeed take place primarily in the target firm and that changes in leadership style are often triggered by changes in organizational structure.
\end{abstract}

Keywords Post-merger integration - Organizational structure · Leadership style · Acquisitions · Manufacturing firms

\section{Einleitung}

Wie verändern sich interne Betriebsabläufe und Struktur eines Unternehmens nach einer Akquisition? Öffentliche Ankündigungen von Fusionen und Übernahmen suggerieren oftmals, dass das Zielunternehmen weitgehend autonom bleibt und dass Synergien durch (oftmals vage spezifizierte) Kosteneinsparungen zustande kommen. Mitarbeiter des Targets sehen Akquisitionen oft mit Skepsis und Unsicherheit, da sie entgegen den öffentlichen Ankündigungen signifikante Änderungen in ihrer Organisation erwarten. Wir adressieren diese Thematik, indem wir die Änderungen im Führungsstil und in der Unternehmensstruktur des akquirierten Unternehmens nach einer Akquisition betrachten.

Die Untersuchung derartiger Veränderungsprozesse ist nicht nur aufgrund der häufig widersprüchlichen öffentlichen Statements interessant. Zum einen wird gescheiterte Post-Merger-Integration (PMI) oft als Hauptgrund dafür genannt, warum Mergers und Acquisitions (M\&As) häufig nicht den erwarteten Wert generieren. ${ }^{1}$ Insbesondere der Transfer von Ressourcen, Kapazitäten und Technologien (Capron 1999; Puranam et al. 2009; Ranft und Lord 2002) und Humanressourcen (Hambrick und Cannella 1993; Larsson und Finkelstein 1999; Schweiger und Weber 1989) sowie inkompatible Unternehmenskulturen (Savović 2017; Li et al. 2018; Larsson und Lubatkin 2001) können den Erfolg von M\&As behindern und trotz hoher potenzieller Synergien zwischen akquirierendem und akquiriertem Unternehmen zu signifikanten Integrationskosten führen (Weber und Schweiger 1992; Weber 2019).

Zum anderen gehen Unternehmen unterschiedlich mit dem Anpassungsprozess nach einer Akquisition um. Während einige einschlägige Arbeiten feststellen, dass

\footnotetext{
1 Wir verwenden in diesem Artikel Fusionen, M\&A und Akquisitionen synonym. Mergers (Fusionen) und Akquisitionen (Übernahmen) unterscheiden sich aus rechtlicher Sicht und in der Außendarstellung als (i) ein „Merger of Equals“ oder einer (ii) ,Top-Down Akquisition“. Unsere Datenbasis beinhaltet Akquisitionen, in denen das akquirierte Unternehmen seine rechtliche Unabhängigkeit verliert. Unsere konzeptionellen Argumente sind jedoch für Mergers und Akquisitionen gleichermaßen gültig.
} 
Käuferunternehmen (Acquirer) dazu tendieren, ihren Führungsstil durchzusetzen (Datta 1991) und die Organisationsstrukturen und -prozesse in den Targets anzupassen (Barkema und Schijven 2008; Björkman et al. 2007), untersuchen Schweiger und Csiszar (2002) ein Produktionsunternehmen, in dem Änderungen nur stark selektiv vorgenommen wurden, sodass der Großteil des Targets davon unberührt blieb. Ähnlich analysiert Schweizer (2005) die Akquisition eines Biotech-Unternehmens durch ein Pharmaunternehmen, in dem die Manager des Zielunternehmens die Freiheit bei der Ausübung ihres Tagesgeschäftes und ihrer Kernaktivitäten beibehielten. Das deutet darauf hin, dass die Organisationsstruktur - wenn überhaupt - nur geringfügig angepasst wurde. Darüber hinaus diskutieren bestehende Arbeiten die Vorteile weniger ,störender“ Koordinationsmechanismen wie der Schaffung einer gemeinsamen Wissens- und Wertebasis als Ersatz für eine strukturelle Integration (Puranam et al. 2009).

Die existierende Forschung zum Erfolg von Mergers and Acquisitions (M\&A) unterteilt den M\&A-Prozess in die Pre-Merger- und die Post-Merger-Phase. Die Literatur zu ersterer konzentriert sich auf die Auswahl des Zielunternehmens, um den Erfolg von Übernahmen zu erklären. Insbesondere wird hier auf den „organisationalen und strategischen Fit" fokussiert, durch den potenzieller Wert generiert wird (Datta 1991; Harrison et al. 1991; Lubatkin 1987; Shelton 1988; Stahl und Voigt 2008; Uhlenbruck et al. 2006). Die empirischen Ergebnisse zum Erfolgsbeitrag von Ex-Ante Fit sind jedoch nicht eindeutig (King et al. 2004). So fokussiert sich ein Teil der Literatur auf die Prozesse nach Übernahme, der Post-Merger Integration (PMI) und deren Beitrag zur Realisierung von Synergien (Cartwright und Schoenberg 2006; Pablo 1994; Rouziès et al. 2019; Trichterborn et al. 2016).

Aus theoretischer Sicht stellen sich eine Reihe von Fragen. Zum einen ist nicht klar, welcher Integrationsgrad ,optimal“ ist, da gewichtige Gründe sowohl für als auch gegen eine umfassende Integration bestehen. Zum anderen ist offen, wie Integrationsprozesse tatsächlich ablaufen: Entsteht aus der Akquisition eine neue Organisation, die entsprechende Anpassungsprozesse vom übernehmenden sowie dem übernommenen Unternehmen erfordert, so dass strukturelle und kulturelle Aspekte der fusionierten Organisation Elemente beider Unternehmensteile ausweist? Besteht ein Machtgefälle zwischen übernehmendem und übernommenem Unternehmen, so dass Änderungen in erster Linie auf Seite des übernommenen Unternehmens stattfinden? Oder führen die meisten Akquisitionen nicht zu tiefgreifenden strukturellen und kulturellen Änderungen? Theoretisch sind alle drei Pfade denkbar, sie hätten jedoch alle unterschiedliche Implikationen für die Implementierung und die zugrundeliegende Motivation von Unternehmensübernahmen. Ausgehend von der Annahme, dass das Machtgefälle zwischen Unternehmen Änderungen im Übernahmeziel wahrscheinlicher macht als im übernehmenden Unternehmen entwickeln wir einen theoretischen Rahmen, den wir anhand einer quantitativ-empirischen Studie überprüfen. Die Bestätigung unseres theoretischen Rahmens liefert Hinweise auf die Existenz und Folgen einer Machtasymmetrie zwischen übernehmenden und übernommenen Unternehmen.

Wir stellen drei Kernhypothesen auf: 
1. Nach einer Akquisition ändern sich sowohl der Führungsstil als auch die Organisationsstruktur eher beim Zielunternehmens als beim Käuferunternehmen bzw. einem nicht-akquirierten Unternehmen.

2. Der Effekt einer Akquisition auf die Veränderung der Organisationsstruktur ist stärker als auf Änderungen des Führungsstils, da er im Gegensatz zum Führungsstil ,top-down“, also durch Anordnung geändert werden kann und weniger auf die freiwillige Kooperation der Mitarbeiter des Zielunternehmens angewiesen ist.

3. Der Einfluss einer Akquisition auf die Veränderung des Führungsstils wird durch die Veränderung der Organisationsstruktur mediiert, da Führungsstile durch die zugrundeliegende Organisationsstruktur des Unternehmens unterstützt werden müssen.

Wir verwenden Daten von 230 europäischen Produktionsunternehmen, um unsere Hypothesen zu testen. Die Wahrscheinlichkeit einer organisationalen Veränderung und einer Änderung des Führungsstils ist für Übernahmeziele höher als für die anderen beiden Gruppen (Übernehmende und nicht-beteiligte Unternehmen), wobei der Effekt auf organisationale Änderungen stärker ist als der auf den Führungsstil. Interessanterweise und entsprechend unserer Hypothese stellen wir fest, dass organisationale Änderungen einen vollständigen Mediationseffekt auf eine Änderung des Führungsstils haben, was darauf hindeutet, dass organisationale Änderungen der Katalysator für eine Änderung des Führungsstils sind. Diese „Sequenz“ der organisationalen Veränderungen ist aufschlussreich: zum einen wird deutlich, dass steuerbare Änderungen wie die Anpassung der Organisationsstruktur auch spontane Änderungen wie eine Änderung des Führungsstils nach sich ziehen kann, was auf die Interdependenzen unterschiedlicher organisationaler Elemente hindeutet (Gulati und Puranam 2009). Zum anderen zeigt sich, dass die oftmals praktizierte Logik, zunächst die Führungskräfte des übernommenen Unternehmens auszutauschen und diese dann strukturelle Änderungen vornehmen zu lassen, möglicherweise unnötige Widerstände im Unternehmen hervorruft.

Wir tragen auf verschiedene Weise zur Literatur bei: Erstens ist unsere Studie eine der wenigen quantitativ-empirischen Studien zum organisationalen Wandel nach Akquisitionen. Zweitens zeigen wir, dass Änderungen in der Organisationsstruktur und im Führungsstil eng miteinander verbunden sind. Dies deutet darauf hin, dass es beim Post-Merger-Integrationsprozess eine logische Abfolge gibt, nach der Änderungen in der Organisationsstruktur einer Änderung im Führungsstil vorausgehen sollten. Dies widerspricht der oft geäußerten Überzeugung von Praktikern, dass die erste Maßnahme der Post-Merger-Integration eine Änderung des Managements des übernommenen Unternehmens sein sollte und das neue Management dann entsprechende strukturelle Änderungen umsetzen sollte.

\section{Post-Merger-Integration - Theorie und Hypothesen}

Die bestehende Forschung zu den Integrationsprozessen nach einer Akquisition ist äußerst umfangreich und soll hier nicht umfassend diskutiert werden. Ein Großteil der bestehenden Studien befasst sich qualitativ mit Integrationsprozessen und 
untersucht insbesondere die Veränderungen im Organisationsdesign und/oder der Unternehmenskultur der beteiligten Unternehmen (Pablo 1994) sowie den Grad der Autonomie, der dem Zielunternehmen gestattet wird (Datta und Grant 1990; Zaheer et al. 2013). Zwei grundlegende, aber nicht notwendigerweise deckungsgleiche Dimensionen der Veränderung sind daher der Grad der (strukturellen und kulturellen) Integration und/oder der Grad der Autonomieaufhebung (Cartwright und Cooper 1993; Haspeslagh und Jemison 1991; Hunt 1990; Nahavandi und Malekzadeh 1988; Shrivastava 1986). Unsere Studie fragt nach den Determinanten der strukturellen und kulturellen Integration und insbesondere nach möglichen Asymmetrien zwischen Käufer- und Zielunternehmen im Rahmen des Integrationsprozesses.

\subsection{Der Hauptort (Locus) der Veränderung bei Integrationsprozessen}

Barkema und Schijven (2008) liefern einen verhaltenstheoretischen Ansatz zur Erklärung von Integrationspraktiken. Sie argumentieren, dass sich begrenzt rationale Käuferunternehmen angesichts der Komplexität der Aufgabe(n), mit denen sie während der Post-Merger-Integration konfrontiert sind, vertraute Lösungen bevorzugen, d.h. sie führen eine „lokale Suche“ durch (Levinthal und March 1993; March 1991). Hier bestimmen drei „Proximitätsregeln“ der Entscheidungsfindung das Such- und Anpassungsverhalten des neu fusionierten Unternehmens: „(1) Suche in der Nähe des Symptoms des Problems und (2) Suche in der Nähe der aktuellen Alternative. (...) (3) Suche in gefährdeten Bereichen des Unternehmens“" (Cyert und March 1963, S. 170-171). Dies impliziert erstens, dass Unternehmen zunächst in den Untereinheiten, die ein Problem melden (,erste Regel“), nach Lösungen suchen. Zweitens werden sie Veränderungen ihrer etablierten Routinen vermeiden, was zu inkrementellen Anpassungen führt (,,zweite Regel“"). Drittens werden Unternehmen Änderungen in weniger leistungsfähigen Untereinheiten implementieren (,dritte Regel“). Im Kontext von M\&As deuten alle drei Proximitätsregeln darauf hin, dass Änderungen in Struktur und Kultur am wahrscheinlichsten in den Targets stattfinden (Barkema und Schijven 2008): Die „,erste Regel“ legt nahe, dass Käufer danach streben werden, Lösungen für Untereinheiten zu finden, da Symptome wie vermehrte Fehlzeiten (Cartwright und Cooper 1990) oder Widerstände bei Mitarbeitern (Larsson und Finkelstein 1999; Nahavandi und Malekzadeh 1988) am wahrscheinlichsten beim Zielunternehmen auftreten. Die ,zweite Regel“ impliziert, dass Käufer versuchen werden, Änderungen innerhalb ihrer eigenen Organisation zu vermeiden, um Schädigungen ihres Status Quos zu minimieren. Letztlich befinden sich Zielunternehmen in einer niedrigeren „Machtposition im System“ (Cyert und March 1963, S. 171). Daher richten sich die Bemühungen um Veränderung eher an die Zielunternehmen, da ihr Widerstandsspielraum limitiert ist. Zusammengefasst lautet unsere erste Hypothese:

Hypothese 1a Die Wahrscheinlichkeit für Veränderungen in der Organisationsstruktur ist bei übernommenen Unternehmen höher als bei Käuferunternehmen.

Neben der Organisationsstruktur haben Unterschiede in den Führungsstilen zwischen übernehmendem und übernommenem Unternehmen in der M\&A-Literatur erhebliche Beachtung gefunden (Datta 1991; Homburg und Bucerius 2006; Savo- 
vić 2017; Larsson und Finkelstein 1999). Die Auswirkungen von M\&As auf den Führungsstil sind besonders interessant, da sie stark von den zugrundeliegenden Organisationskulturen beeinflusst werden und im Vergleich zu Organisationsstrukturen möglicherweise schwieriger zu ändern sind (Buono und Bowditch 1989; Chatterjee et al. 1992). Zum Beispiel lässt sich Führungsstil weniger leicht durch bloße „Anordnung“ ändern.

Wir argumentieren, dass ,das Management der Käuferunternehmen letztlich dem Management der übernommenen Unternehmen ihren eigenen [Führungs-]Stil auferlegt“" (Datta 1991, S. 284; eigene Übersetzung). Zudem ist zu erwarten, dass die Proximitätsregeln von Cyert und March (1963) auch für potenzielle Veränderungen im Führungsstil gelten. Folglich lautet die Hypothese 1b bezüglich Änderungen der Führungsstile im Kontext von M\&As:

Hypothese 1b Die Wahrscheinlichkeit für Veränderungen im Führungsstil ist bei übernommenen Unternehmen höher als bei Käuferunternehmen.

\subsection{Eine Schockreaktion?}

Die bestehende Forschung zu Integrationsprozessen nach Akquisitionen hat insbesondere Käufer- und übernommene Unternehmen in ihrem Verhalten und dessen Konsequenzen verglichen. Wir erweitern die Analyse, indem wir Unternehmen, die an keinen M\&A-Transaktionen beteiligt waren (nicht-fusionierende Unternehmen) als Kontrollgruppe verwenden, um der Frage nachzugehen, ob Veränderungen und deren Herausforderungen ein Grund für den oftmals ernüchternden Erfolg von Akquisitionen sein können.

Die unternehmensweiten Ergebnisse von M\&As wurden häufig mit nicht fusionierenden Unternehmen verglichen: Beispielsweise untersuchen die meisten Studien zum strategischen oder organisationalen Fit von M\&As hauptsächlich den Einfluss auf abnormale Renditen und vergleichen somit die Aktienkursentwicklung von Käufern mit denen anderer (weder auf der akquirierenden noch auf der akquirierten Seite einer Fusion beteiligten) Unternehmen (Capron und Pistre 2002; Chatterjee 1992; Kim und Finkelstein 2009; Singh und Montgomery 1987; Uhlenbruck et al. 2006). Falls Ergebnisse jedoch mit nicht-fusionierenden Unternehmen verglichen werden und die meisten Änderungen der Organisation und Führung in Zielunternehmen auftreten (die wiederum den Erfolg der Akquisition an sich beeinflussen können), müssen die Veränderungsprozesse von Zielunternehmen auch mit nicht-fusionierenden Unternehmen verglichen werden.

Gegeben unserer Argumentation, dass es zu einer höheren Wahrscheinlichkeit von Veränderungen im Zielunternehmen kommt, die durch den ,Schock“ der Übernahme entstehen (ein Schock, der nicht-fusionierende Unternehmen nicht betrifft), lauten unsere Hypothesen 2a und $2 \mathrm{~b}$ daher:

Hypothese 2a Die Wahrscheinlichkeit für Veränderungen in der Organisationsstruktur ist bei übernommenen Unternehmen höher als bei nicht-fusionierenden Unternehmen. 
Hypothese 2b Die Wahrscheinlichkeit für Veränderungen im Führungsstil ist bei übernommenen Unternehmen höher als bei nicht-fusionierenden Unternehmen.

\subsection{Die Beziehung zwischen Unternehmensstruktur und Führungsstil}

Es liegt auf der Hand, dass Änderungen in der Organisationsstruktur und im Führungsstil miteinander verbunden sind, insbesondere, da zeitweise Inkonsistenzen zwischen der formalen Organisation, dem bewusst ,entworfenen normativen System“, und der informellen Organisation, den ,organisationsintern auftretenden Pfaden der sozialen Interaktion“ ein potenzieller Grund dafür sind, dass M\&As oftmals keinen Wert generieren (Gulati und Puranam 2009, S. 422; eigene Übersetzung). Organisationen, die sich im Prozess der Neukonfiguration oder Umstrukturierung befinden, entstehen als Ergebnis solcher Inkonsistenzen häufig erhebliche Kosten (Amburgey et al. 1993; Lamont et al. 1994; Ceci et al. 2016; Oxman und Smith 2003).

Die Unternehmenskultur hat einen starken Einfluss auf den Führungsstil, inklusive der Art und Weise, wie Manager untereinander und mit den ihnen untergeordneten Personen interagieren. Anpassungen des Führungsstils setzen daher Änderungen in den zugrundeliegenden Werten und Überzeugungen voraus (Datta 1991; Lorsch 1986; Schein 1985; Sørensen 2002). Hingegen können Änderungen in der formalen Organisation schneller implementiert werden (Lamont et al. 1994; Nickerson und Zenger 2002). Werden also beide Prozesse simultan durch eine Akquisition ausgelöst, können temporäre Inkonsistenzen entstehen. Aufgrund der Herausforderungen bei der Implementierung sowie der Geschwindigkeit von Änderungen in Unternehmensstruktur und Führungsstil lautet Hypothese 3:

Hypothese 3 Die Wahrscheinlichkeit einer Veränderung der Organisationsstruktur in übernommenen Unternehmen ist höher als einer Veränderung des Führungsstils in übernommenen Unternehmen.

Obwohl Arbeiten über den Effekt verschiedener Führungsstile bei M\&As existieren (Datta 1991; Larsson und Finkelstein 1999), gibt es nur wenig Forschung über die Instrumente, mit denen eine Konvergenz im Führungsstil erreicht werden kann (außer natürlich dem Ersetzen der Führungskräfte des Zielunternehmens (Lubatkin et al. 1999).

Einen wichtigen Kanal für die Änderung des Führungsstils bilden die Werte und Überzeugungen eines Unternehmens. Insbesondere durch die „Änderung des Namens des Zielunternehmens sowie seiner formalen Struktur, (...), kann der Käufer versuchen dem Zielunternehmen seine Überzeugungen, Annahmen und Werte aufzuzwingen“ (Larsson und Lubatkin 2001, S. 1578; eigene Übersetzung). Ähnlich hierzu fand Schweizer (2005), der Akquisitionen kleiner Biotech-Unternehmen durch große Pharmakonzerne analysierte, dass den jungen Unternehmen die formale Struktur eines großen Unternehmens aufgebürdet wurde. Dies führte zu substanziellen Änderungen der Unternehmenskultur der Targets führte und beeinflusste dabei die firmenspezifische unternehmerische Kultur. 
Verschiedene Mechanismen können zur Anpassung des Führungsstils nach strukturellen Veränderungen führen: So kann eine Veränderung der Struktur beispielweise zu einer Veränderung der Kontrollspanne führen, wodurch wiederum andere Führungsinstrumente optimal werden. Gleichermaßen können strukturelle Veränderungen den Fokus auf andere Handlungsfelder richten, so dass Entscheidungsträger diese veränderten Prioritäten in ihren Werten und demnach ihrem Führungsstil widerspiegeln. Schließlich ist es denkbar, dass strukturelle Veränderungen auch personelle Wechsel nach sich ziehen und dass die neu ausgewählten Führungskräfte einen anderen, dem Käuferunternehmen näherstehenden Führungsstil pflegen.

Daher können Veränderungen in der Unternehmensstruktur des Targets das Verhältnis zwischen Akquisition und einer Änderung des Führungsstils mediieren (Venkatraman 1989). Die Werte und Überzeugungen eines Unternehmens beeinflussen den Führungsstil und gleichzeitig können diese Werte und Überzeugungen bei einer Akquisition von außen durch strukturelle Änderungen beeinflusst werden. Daher könnte der Effekt von Akquisition auf Änderungen im Führungsstil zumindest teilweise auf Änderungen in der Unternehmensstruktur zurückzuführen sein. Folglich könnten Käufer, die ihren Zielunternehmen Änderungen in der Unternehmensstruktur aufzwingen, indirekt Veränderungen im Führungsstil des Zielunternehmens auslösen. Folglich lautet Hypothese 4:

Hypothese 4 Die Wahrscheinlichkeit einer Änderung des Führungsstils in einem übernommenen Unternehmen wird durch eine Änderung in der Organisationsstruktur mediiert.

\section{Methodik}

Empirische Forschung zu M\&As stützte sich bisher auf drei Arten von Datenquellen: öffentlich verfügbare Daten wie Aktien-, Buchhaltungs- oder Branchenklassifizierungsinformationen (Ramaswamy 1997; Shelton 1988; Uhlenbruck et al. 2006), veröffentlichte Fallstudien (Larsson und Finkelstein 1999) oder Umfragen (Capron et al. 1998; Chatterjee et al. 1992; Datta 1991). Für Post-Merger-Prozesse scheinen Umfragen am geeignetsten (Capron 1999; Zaheer et al. 2013). Um größeres Detailwissen und hohe Rücklaufquoten zu erhalten, wurde für diese Studie ein Team aus zwölf studentischen Interviewern engagiert, das detaillierte Telefoninterviews durchführte. Vor Beginn des Projekts wurden die Interviewer bezüglich des Inhalts und der Methodik intensiv geschult. Während der Interviewphase erhielten sie fortlaufend Feedback zu ihren Interviews über Inhalt und Vorgehen. Alle Interviews wurden von Muttersprachlern durchgeführt. 


\subsection{Stichprobengrundlage}

Wir befragten 230 österreichische, deutsche, spanische und schweizer Produktionsunternehmen, um unsere Hypothesen zu testen. ${ }^{2,3}$ Käufer und Zielunternehmen wurden aus der M\&A-Datenbank der Universität St. Gallen sowie der SCD-Datenbank von Thomson Reuters identifiziert. Während sich die erste Datenbank insbesondere auf Transaktionen im D/A/CH Raum konzentriert, bietet die zweite Datenbank weltweite Informationen zu M\&As. Eine Transaktion wurde nur in die Stichprobe der zu kontaktierenden Unternehmen aufgenommen, wenn Käufer und Zielunternehmen noch identifizierbar waren, z. B. wenn das übernommene Unternehmen ein Geschäftsbereich des Käufers wurde. Zunächst wurde das Käuferunternehmen kontaktiert und der Interviewpartner nach einem abgeschlossenen Interview nach Ansprechpersonen gefragt, die dem übernommenen Unternehmen angehört hatten. Die nicht fusionierenden Unternehmen wurden zufällig aus AMADEUS ausgewählt, einer von Bureau von Dijk angebotenen Datenbank, die Finanzinformationen sowie allgemeine Unternehmenscharakteristika wie eine Branchenklassifikationen für europäische Unternehmen enthält. ${ }^{4}$ Wir haben Spanien als viertes Land ausgewählt, um mögliche Besonderheiten der deutschsprachigen Märkte zu ermitteln und ggf. den Geltungsbereich unserer Hypothesen und Ergebnisse einzuschränken.

Wir haben uns auf Produktionsunternehmen konzentriert, um Konsistenz zu gewährleisten, um die Heterogenität in der Grundstruktur der Unternehmen und deren Produktionsprozesse zu verringern. Darüber hinaus konnten wir hierdurch die individuellen Produktionsstätten zwischen den ehemaligen Teilen des Acquirers und Targets unterscheiden. Wir haben M\&As aus den Jahren 2007-2010 ausgewählt, sodass die Änderungen nach der Akquisition genügend Zeit hatten, sich zu entfalten.

Die Interviewer kontaktierten 825 Käufer, 690 Zielunternehmen und 308 nichtfusionierende Unternehmen in zwei Erhebungswellen. Daraus resultierten 229 Interviews mit Käufern, 169 Interviews mit Zielunternehmen (die gemäß der Kaskadenmethode bei der Gewinnung neuer Interviewpartner von einem der erfolgreich interviewten Käuferunternehmen übernommen wurden) und 103 Interviews mit nicht fusionierenden Unternehmen. Dies entspricht Rücklaufquoten von $28 \%$ für Käufer, $24 \%$ für Zielunternehmen und 33\% für nicht fusionierende Unternehmen, vergleichsweise hohe Quoten im Vergleich zu Studien zu verwandten Themen (Capron 1999; Zaheer et al. 2013).

\footnotetext{
2 Bei zehn Beobachtungen wurde nur ein Geschäftsbereich gekauft. Der Ausschluss dieser Beobachtungen ändert nichts an den Ergebnissen.

3 Da Spanien stärker unter der Finanzkrise gelitten hat als die deutschsprachigen Länder, haben wir unser Modell ohne die spanischen Beobachtungen neu geschätzt. Die Ergebnisse sind vergleichbar mit den Ergebnissen für die vollständige Stichprobe. Nur der Effekt in Bezug auf Hypothese $1 \mathrm{~b}$ wurde nicht signifikant. Angesichts der Tatsache, dass wir 22\% der Acquirer und 19\% der Targets verlieren, wenn wir die spanischen Beobachtungen ausschließen, haben wir nach dem Zufallsprinzip die gleiche Anzahl von Acquirern und Targets unabhängig von ihrer Nation ausgeschlossen und festgestellt, dass die Hypothese 1b erneut nicht signifikant wird. Dies legt nahe, dass das Ergebnis in Bezug auf Hypothese 1b eher sensitiv auf die Stichprobengröße als auf den Ausschluss Spaniens reagiert.

${ }^{4}$ Bei der zufälligen Auswahl der nicht fusionierenden Unternehmen folgen wir Krug und Hegarty (1997), wobei wir die Fluktuation im Management zwischen übernommenen und nicht fusionierenden Unternehmen vergleichen.
} 


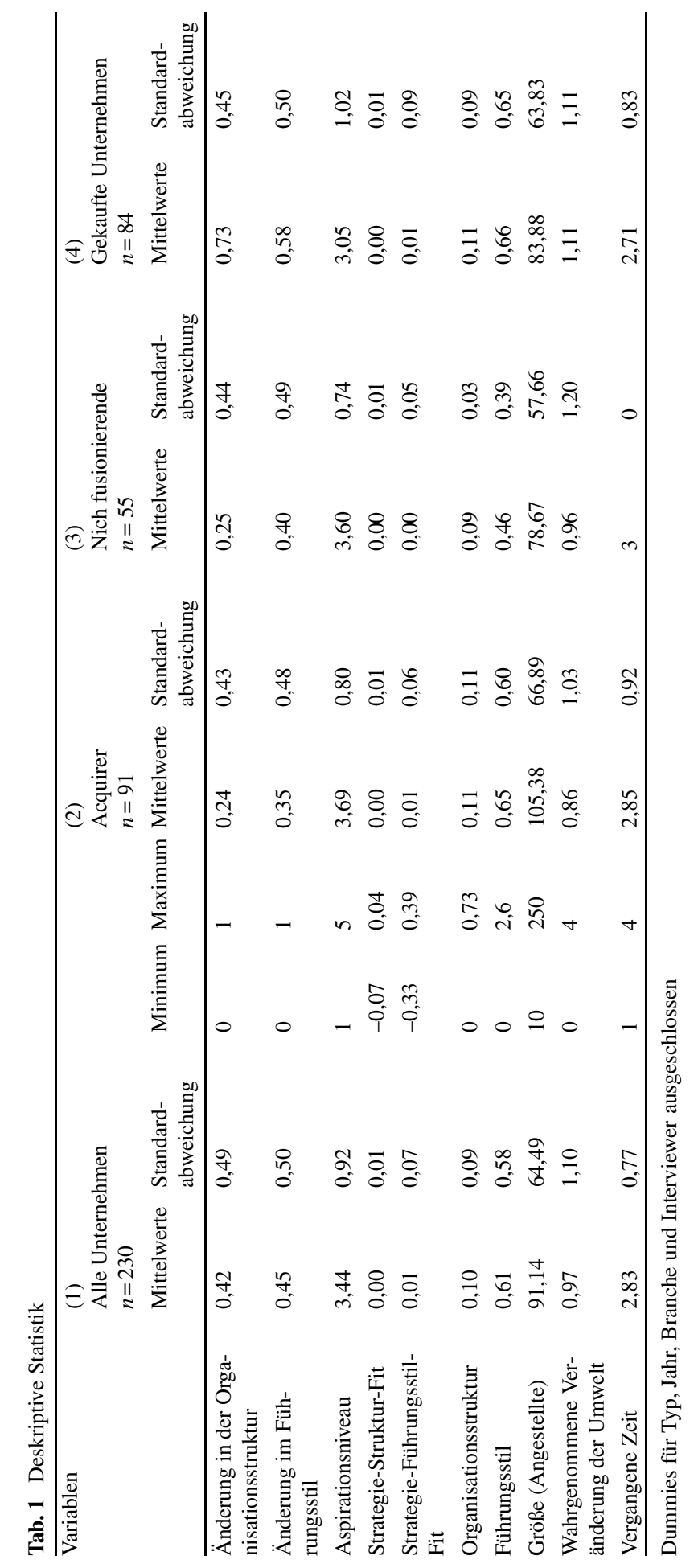




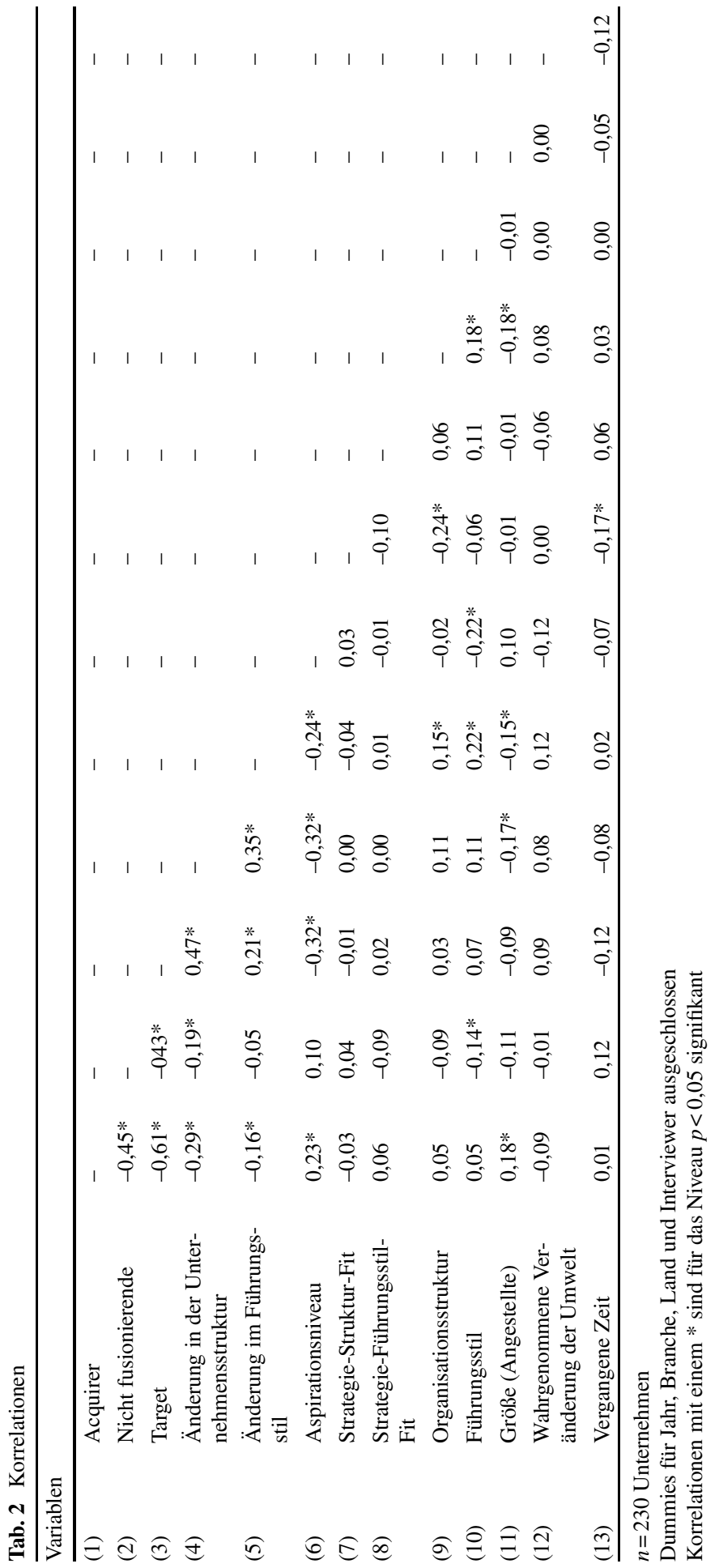


Die Interviewpartner waren hauptsächlich mittlere Führungskräfte, insbesondere Produktionsmanager. Dies hatte zwei Vorteile: Zum einen können mittlere Führungskräfte gute Einblicke in den Führungsstil sowie die Unternehmensstruktur geben, zum anderen mussten die Interviewpartner in der Lage sein, die stattfindenden Änderungen zu bewerten, sollten jedoch nicht direkt in den Entscheidungsprozess involviert sein, der zur Transaktion führte, um den Effekt der Ex-Post-Rationalisierung zu verringern. Wenn keine mittleren Führungskräfte verfügbar waren, wurden Umfrageteilnehmer aus angrenzenden Führungsebenen befragt. ${ }^{5}$

Die Unternehmen in unserer Stichprobe hatten zum Zeitpunkt der Transaktion zwischen 10 und 250 Mitarbeiter. Diese Einschränkung erhöht die Repräsentativität und Genauigkeit der Antworten und reduziert mögliche Verzerrungen aufgrund von Größeneffekten. Die befragten Standorte waren in mehr als $75 \%$ der Interviews zum Zeitpunkt der Transaktion entweder der Hauptsitz oder eine Produktionsstätte.

Käufer und Zielunternehmen wurden nach verschiedenen organisationalen Merkmalen in den drei Jahren vor der Transaktion und nach der Transaktion befragt. Die identischen Items wurden für nicht fusionierende Unternehmen verwendet. Da sie jedoch nicht an einer Fusion beteiligt waren, wurden sie nach der Situation in den drei Jahren vor dem Jahr 2007 (zwischen 2004 und 2007) und nach dem Stand seitdem gefragt. Wir haben das Jahr 2007 gewählt, da dies das Jahr war, in dem sich die Auswirkungen der Wirtschaftskrise zu entfalten begannen. Wir erwarteten daher, dass die Befragten besonders sensitiv auf seitdem stattgefundene Veränderungen reagierten. Im Durchschnitt waren seit den M\&As knapp drei Jahre $(2,8)$ vergangen, sodass ausreichend Zeit für die Implementierung von Änderungen war. Bei der Befragung der nicht-fusionierenden Unternehmen im Jahr 2010 betrug die Zeitspanne für Änderungen ebenfalls drei Jahre.

Die meisten Umfrageitems, die zur Messung der organisationalen Merkmale verwendet wurden, waren aus früheren Untersuchungen abgeleitet und an den spezifischen Kontext angepasst. Teile der Erhebungsinstrumente wurden 2008 bei zwei früheren Datenerfassungswellen mit über 1000 Befragten verwendet (Mahr 2010). Vor den zwei Wellen im Jahr 2008 wurden die Umfrageergebnisse mit Produktionsmanagern diskutiert und es fand ein Pretest mit 15 Interviews statt.

Die Interviewer sicherten den Befragten Anonymität und Vertraulichkeit zu, um die Tendenz zur sozialen Erwünschtheit zu verringern (Chang et al. 2010). Darüber hinaus wurden die Informationen, die den wichtigsten unabhängigen Variablen (Unternehmenstypen - Käufer, nicht fusionierende Unternehmen und übernommene Unternehmen) zugrunde liegen, nicht während der Umfrage erfasst, sondern aus zwei M\&A-Datenbanken gewonnen (Capron und Guillén 2009), um potenziellen „common method bias“ zu verringern (Podsakoff et al. 2003).

\footnotetext{
5 Die Auswahl der alternativen Interviewpartner war pragmatisch bedingt. Wir denken, dass durch die Interviews mit Mitarbeitern aus angrenzenden Hierarchieebenen zwar Messfehler auftreten können, aber keine systematischen Verzerrungen. Die empirischen Modelle wurden auch unter Ausschluss der, alternativen" Interviewpartner geschätzt. Die Ergebnisse sind qualitativ gleich (wenn auch statistisch weniger signifikant, da die Stichprobengröße reduziert wird).
} 
Die zur Messung und Berechnung der verschiedenen Variablen verwendeten Items befinden sich im Anhang. Deskriptive Statistiken und Korrelationen für die einzelnen Kennzahlen sind in Tab. 1 und 2 wiedergegeben. ${ }^{6}$

\subsection{Abhängige Variablen}

\subsection{1 Änderung in der Organisationsstruktur}

Die Änderung der Organisationsstruktur wird mit einer Dummyvariable gemessen, die eine Änderung des Dezentralisierungsgrades der Entscheidungsrechte innerhalb der Unternehmen anzeigt. Der Grad der Dezentralisierung ist eine Schlüsselkomponente der Organisationsstruktur und ein wichtiger Hebel für Veränderungsprozesse (Nickerson und Zenger 2002; Phan und Hill 1995; Pugh et al. 1968). Die Befragten wurden darum gebeten, anzugeben, auf welcher Ebene innerhalb ihrer Organisationshierarchie unterschiedliche Entscheidungen getroffen wurden. ${ }^{7}$ Wir haben die einzelnen Items aus der bestehenden Forschung zur Dezentralisierung übernommen und angepasst (Andersen und Jonsson 2006; Bloom et al. 2008; Burton et al. 2002; Hanks et al. 1993; Lee und Grover 2000; Nahm et al. 2003; Pugh 1973). Für jede der einzelnen Entscheidungen gaben die Manager an, auf welcher Hierarchieebene die Entscheidung vor und nach den M\&As getroffen wurde. Darüber hinaus wurden die Befragten nach der Gesamtzahl der Hierarchieebenen vor und nach der Transaktion gefragt.

Auf dieser Basis haben wir den relativen Dezentralisierungsgrad für jedes Entscheidungsrecht berechnet, indem wir die Hierarchieebenen der einzelnen Entscheidungsrechte durch die Gesamtzahl der Hierarchieebenen im relevanten Zeitraum dividiert haben. Diese Werte wurden standardisiert, wobei der Wert 1 vollständige Dezentralisierung und 0 vollständige Zentralisierung bedeutet. Schließlich haben wir für die sechs Entscheidungsrechte, die zur Bestimmung des Dezentralisierungsgrades in den Unternehmen verwendet wurden (Cronbachs $\alpha=0,81$ ) die Änderungen zwischen den beiden Zeithorizonten berechnet. Hierfür haben wir den Dummy „Änderung der Organisationsstruktur" erstellt, der den Wert 1 annimmt, wenn bezüglich mindestens einer der sechs relevanten Entscheidungsrechte eine Änderung stattgefunden hatte.

\subsection{2 Änderung im Führungsstil}

Die Änderung des Führungsstils wird ebenfalls als Dummy gemessen, der eine Änderung des Grads der Mitarbeiterbeteiligung anzeigt (Datta 1991; Vroom und Yetton 1973). Wir konzentrieren uns auf diese Dimension des Führungsstils, da sie konzeptionell vergleichbar zur formalen Verteilung von Entscheidungsrechten ist. Die relevanten Items wurden auf der Grundlage der vorhandenen Literatur zur Beteiligung an Entscheidungsprozessen entwickelt (Coyle-Shapiro 1999; Dachler

\footnotetext{
6 Wir werden uns manchmal auf zwei Zeithorizonte, nämlich vor und nach dem M\&A beziehen. Für nicht fusionierende Unternehmen bedeutet dies ,,vor 2007“ und ,nach 2007““.

7 Die Fragen sind im Anhang aufgeführt.
} 
und Wilpert 1978; Gallie 2013; Heller 1977; Long 1981; Ruh et al. 1975; Schuler 1977; Vroom 1959). Die Befragten sollten auf einer Likert-Skala von („1 - trifft überhaupt nicht zu“) bis (,,5 - trifft voll und ganz $\left.\mathrm{zu}^{\prime \prime}\right)$ angeben, in welchem Maße sie unterschiedlichen Aussagen zur Beteiligung der (Produktions-)Mitarbeiter an Entscheidungsprozessen innerhalb ihrer Organisation zustimmten. Erfragt wurden Aspekte wie die allgemeine Offenheit der Kommunikation zwischen Managern und Produktionsmitarbeitern oder der Grad, zu dem Produktionsmitarbeiter Verbesserungsvorschläge machen können. Die Befragten gaben erneut Bewertungen für die Zeiträume vor und nach den M\&As ab. Wie der Dummy ,Änderung der Organisationsstruktur“ nimmt der Dummy „Änderung des Führungsstils“ den Wert 1 an, wenn mindestens eine Änderung in einem der fünf Items zur Messung des Partizipationsgrades stattgefunden hatte $(\alpha=0,84)$.

Obwohl beide Variablen verhältnismäßig sensitiv auf kleine Änderungen scheinen, halten wir diese Operationalisierung aus zwei Gründen für geeignet: Erstens können schon kleine Anpassungen von Mitarbeitern als scherwiegende Veränderungen wahrgenommen werden (Cartwright und Cooper 1990; Schweiger und Ivancevich 1985) und starke Reaktionen hervorrufen, wenn ihre Erwartungen beeinflusst werden oder die Änderungen als gravierend empfunden werden. Zweitens haben nur $24 \%(37 \%)$ der nicht gekauften Unternehmen eine Änderung der Organisationsstruktur (Führungsstil) erfahren, obwohl die Variablen auf jede Änderung in Bezug auf die sechs bzw. fünf Items reagieren. Übermäßige Sensitivität stellt daher hier kein Problem dar. In einer Robustheitsanalyse verwenden wir das tatsächliche Ausmaß in einem linearen OLS-Modell und erhalten vergleichbare Ergebnisse.

\subsection{Unabhängige Variablen}

\subsubsection{Unternehmenstypen}

Wir unterscheiden drei Arten von Unternehmen anhand von Dummy-Variablen: Käufer, übernommene Unternehmen und nicht fusionierende Unternehmen. Die Informationen zum Unternehmenstyp stammen aus den oben beschriebenen M\&ADatenbanken. Sofern nicht anders angegeben, verwenden wir die übernommenen Unternehmen als Referenzgruppe.

\subsection{Kontrollvariablen}

Wir kontrollieren eine Reihe von internen (Aspirationsniveau, strategischer Fit, Trägheit) und externen Faktoren (wahrgenommene Turbulenz der Umwelt, Industrie-, Jahres- und Ländereffekte), die Veränderungsaktivitäten in Organisationen beeinflussen können.

\subsubsection{Aspirationsniveau}

Die Behavioral Theory of the Firm hat die Rolle der organisationalen Performance und das Erreichen von Aspirationsniveaus für Veränderungsprozesse hervorgehoben (Greve 1998; Labianca et al. 2009). Die fundamentale Logik besteht darin, 
dass Unternehmen Aspirationen hinsichtlich ihrer Performance entwickeln und ihre tatsächliche Performance mit diesen Aspirationen vergleichen. Abhängig vom Erreichen ihrer Ziele steigt die Risikobereitschaft und damit die Wahrscheinlichkeit organisationaler Veränderungen (Grohsjean et al. 2015).

Das Erreichen des Aspirationsniveaus der Organisationen wird anhand des Durchschnitts zweier Likert-Skalen $(\alpha=0,69)$ gemessen. Die Befragten wurden gebeten, auf einer Skala von , 1 - trifft überhaupt nicht zu“ bis ,,5 - trifft voll zu“ zu bewerten, ob die Gesamtleistung des Unternehmens ihre Erwartungen erfüllt hatte und ob die Performance des Unternehmens die Performance ihrer wichtigsten Wettbewerber in der Zeit vor den M\&As überstieg.

\subsubsection{Strategischer Fit}

Das Konzept des „Fits“ zwischen den organisationalen Elementen eines Unternehmens und seiner Strategie ist in der Literatur etabliert (Chandler 1962; Venkatraman und Camillus 1984; Yin und Zajac 2004). Analog dazu muss der Führungsstil - inklusive des Kommunikationsverhaltens und damit des Partizipationsgrades innerhalb des Unternehmens - zur Strategie eines Unternehmens passen (Benner und Tushman 2003; Bauer et al. 2018; Miller 1987; Roberts 2004). Unternehmen, deren Organisationsdesign und Strategie sich nicht gegenseitig verstärken, sind weniger erfolgreich, was den Druck erhöht, die Organisationsstruktur und den Führungsstil an ihre Strategie anzupassen (Burton et al. 2002; Donaldson 1987).

Wir haben den Dezentralisierungs-Strategie-Fit sowie den Partizipations-Strategie-Fit der Unternehmen vor der Transaktion gemessen. Dazu haben wir den jeweiligen Dezentralisierungsgrad bzw. Partizipationsgrad vor der Transaktion mit den PreM\&A-Strategien der Unternehmen multipliziert. Die Pre-M\&A-Strategie wurde anhand der ,relativen Explorationsorientierung“ gemessen. Dezentralisierung und Partizipation wurden berechnet, indem die Mittelwerte der Pre-M\&A-Werte der Items für Dezentralisierung und Partizipation ermittelt wurden, diese wurden dann durch Subtraktion des jeweiligen Medians standardisiert. Positive Werte weisen daher auf einen relativ hohen, negative Werte auf einen geringen Grad an Dezentralisierung und Partizipation hin. Die einzelnen Items sind im Anhang aufgeführt. ${ }^{8}$

Wir haben den Grad des Fits in der Zeit vor den M\&As berechnet, indem wir die standardisierten relativen Explorationswerte mit den standardisierten Dezentralisierungs- und Partizipationsgraden multipliziert haben, was zu positiven ,Strategischen Fit“-Werten für hohe relative Exploration/hohe Dezentralisierung, hohe relative Exploration/hohe Partizipation, geringe relative Exploration/geringe Dezentralisierung und geringe relative Exploration/geringe Partizipation führte. ${ }^{9}$

\footnotetext{
8 Siehe auch Dehne (2013) für mehr Details zur Datenerhebung.

9 Das folgende Beispiel beschreibt unser Vorgehen: Ein Unternehmen mit einem positiven Explorationswert (z. B. +2) erreicht den genannten Wert mit einem gleichzeitig positiven (über dem Durchschnitt liegenden Median) Partizipations-/Dezentralisierungwert (z. B. +3), da das Ergebnis der Multiplikation positiv ist (hier: +6). Genauso kann ein Unternehmen einen positiven Wert erreichen, wenn ein negativer relativer Explorationswert (d.h. Fokus auf Exploitation) (von z.B. -3) mit einem (passenden) negativen Partizipations-/Dezentralisierungswert (von z.B. -2) kombiniert wird. Dies führt wieder zu einem Wert
} 


\subsubsection{Organisationsstruktur/Führungsstil}

Strukturelle und organisationale Trägheit können die Bereitschaft zu Veränderungen in Unternehmen verringern (Hannan und Freeman 1984; Kelly und Amburgey 1991). Wir nehmen an, dass sich Institutionen und Routinen umso leichter entwickeln und die Trägheit bezüglich Veränderungen erhöhen, je mehr sich Unternehmen im Hinblick auf ihre Organisationsstruktur (Dezentralisierung) und ihren Führungsstil (Partizipation) auf eine klare „Richtung“ fokussiert haben. Der Grad der Dezentralisierung vor den M\&As wird berechnet, indem der Mittelwert der Pre-M\&A-Werte der sechs Dezentralisierungsitems verwendet wird, anhand derer wir die Änderung in der Organisationsstruktur ermittelt haben. Diese Werte wurden standardisiert, indem die absolute Abweichung vom Median dieser Variable berechnet wurde. Dadurch sind die Werte umso höher, je stärker oder schwächer die Unternehmen vor der Transaktion dezentralisiert waren. Wir haben denselben Vorgang verwendet, um den anfänglichen Partizipationsgrad für die Unternehmen zu berechnen.

\subsubsection{Größe}

Die Größe der Organisation kann ebenso die Trägheit des Unternehmens erhöhen (Hannan und Freeman 1984). Wenn Unternehmen wachsen, legen sie Wert auf Effizienz, Formalisierung und Vorhersehbarkeit, was zu Inflexibilität führt und Veränderungen behindert (Quinn und Cameron 1983). Wir verwenden den natürlichen Logarithmus der Anzahl der Mitarbeiter am Standort zum Zeitpunkt der Transaktion als Proxy für die Größe.

\subsubsection{Umweltfaktoren}

Neben den internen Faktoren sind auch Umweltfaktoren eine wichtige Determinante für das Organisationsdesign (Burton et al. 2002; Miller 1992). Nickerson und Zenger (2002) konzentrieren sich auf Veränderungsprozesse in der Organisationsstruktur und erwähnen, dass ,strukturellen Veränderungen [...] Veränderungen in der Umwelt folgen müssen“ (S. 547, eigene Übersetzung). Wir kontrollieren sowohl objektive als auch subjektiv wahrgenommene Veränderungen der Umwelt. Die subjektiv wahrgenommenen Veränderungen der Umwelt werden durch ein Item gemessen, das die Stabilität der Umwelt erfasst. Die Befragten wurden gebeten, für die beiden Zeithorizonte vor und nach den M\&As auf einer Likert-Skala von ,1 - sehr unsicher“ bis ,,5 - sehr stabil“ anzugeben, wie sie die Stabilität ihrer Umgebung wahrnahmen, wobei darunter ihre Kunden, Wettbewerber, Technologien, Zulieferer, Kapitalmärkte sowie Staat und Gesellschaft fielen. Wir berechnen die absolute Differenz zwischen beiden Zeithorizonten, um den Grad der wahrgenommenen Umweltveränderung zu bewerten. Um für „objektive“ Umweltunterschiede zu kontrollieren, schließen wir Dummies für Industrie (zweistellige SIC-Codes), Land und Jahr (der M\&As) ein.

von +6 . Wohingegen ein Unternehmen mit positiven Partizipations-/Dezentralisierungswert (z. B. +2) zu einem negativen Wert von -6 führt. 


\subsubsection{Zusätzliche Kontrollvariablen}

Wir nehmen zwei zusätzliche Variablen in unsere Schätzungen auf: die Zeit seit der Transaktion und Interviewereffekte. Es kann einige Zeit dauern, bis sich Änderungsprozesse entfalten. Acquirer warten möglicherweise eine gewisse Zeit, bis sie größere Änderungen vornehmen (Barkema und Schijven 2008; Graebner et al. 2017; Teerikangas und Thanos 2018; Lamont et al. 1994; Schweiger et al. 1987). Wir schließen daher eine Kontrollvariable für die seit den M\&As vergangene Zeit ein. Schließlich verwenden wir trotz des hohen Standardisierungsgrades der verwendeten Items Interviewer-Dummies, um für mögliche Heterogenität zwischen Interviewern zu kontrollieren.

\subsection{Schätzmodelle}

Die in dieser Studie analysierten abhängigen Variablen sind dichotom - Änderungen in der Organisationsstruktur und im Führungsstil werden als „,1“ codiert, wenn eine Änderung stattgefunden hat, und als , $0^{“}$ falls nicht. Demnach verwenden wir ein Logit-Modell, um Effekte auf die Änderungswahrscheinlichkeit der Organisationsstruktur und des Führungsstils zu analysieren. Darüber hinaus konzentrieren sich unsere Analysen eher auf die marginalen Effekte als auf die Regressionskoeffizienten, da diese ein genaueres Bild von der Beziehung zwischen den abhängigen und den unabhängigen Variablen vermitteln (Hoetker 2007; Wiersema und Bowen 2009). Die marginalen Effekte werden als durchschnittliche marginale Effekte berechnet, d.h. sie zeigen für die relevante Variable den Durchschnitt der marginalen Effekte aller Beobachtungen an. So können wir die marginalen Effekte, die wir zum Testen von Hypothese 3 benötigen, modellübergreifend vergleichen (Mood 2010).

Wir schätzen die folgenden Regressionen zum Testen unserer Hypothesen:

$$
\begin{aligned}
& \operatorname{Prob}\left(\left(\text { OrgStrukÄnd }_{i}\right)=1\right)= \\
& F\left(\beta_{0}+\beta_{1} \cdot \text { Acquirer }_{i}+\beta_{2} \cdot \operatorname{NichtFus}_{i}+\beta_{3} \cdot \operatorname{AspNiv}_{i(t-1)}+\beta_{4} \cdot\right. \\
& \text { StratStrukFit }_{i(t-1)}+\beta_{5} \cdot \operatorname{OrgStruk}_{i(t-1)}+\beta_{6} \cdot \operatorname{Größe}_{i(t-1)}+\beta_{7} \cdot \\
& \text { WahrnUmw }_{i}+\beta_{8} \cdot \text { ZeitVerg }_{i}+\beta_{9-n} \cdot\left[\text { Kontrollvariablen }_{i}+\varepsilon_{i}\right) \\
& \operatorname{Prob}\left(\left(\text { FührStilÄnd }_{i}\right)=1\right)= \\
& F\left(\beta_{0}+\beta_{1} \cdot \text { Acquirer }_{i}+\beta_{2} \cdot \operatorname{NichtFus}_{i}+\beta_{3} \cdot \operatorname{AspNiv}_{i(t-1)}+\beta_{4} \cdot\right. \\
& \text { StratFührStileFit }_{i(t-1)}+\beta_{5} \cdot \text { FührStil }_{i(t-1)}+\beta_{6} \cdot \operatorname{Größe}_{i(t-1)}+\beta_{7} \cdot \\
& \text { WahrnUmw }_{i}+\beta_{8} \cdot \text { ZeitVerg }+\beta_{9-n} \cdot\left[\text { Kontrollvariablen }_{i}+\varepsilon_{i}\right)
\end{aligned}
$$

Hierbei steht „,i“ für die einzelnen Unternehmen und „(t-1)“ für den Zeitraum vor den M\&As/vor 2007. 


\section{Ergebnisse}

\subsection{Kernergebnisse}

Tab. 3 enthält die logistischen Regressionen für die Hypothesen 1 und 2. Für beide abhängigen Variablen haben wir zunächst Modelle nur mit Kontrollvariablen geschätzt: Modell 1 für die Änderung der Organisationsstruktur und Modell 3 für die Änderung des Führungsstils. Das Hinzufügen der Dummies für Käufer und nicht fusionierende Unternehmen zur Unterscheidung der verschiedenen „Unternehmenstypen“ (Modell 2 für die Änderung der Organisationsstruktur und Modell 4 für die Änderung des Führungsstils) erhöht in beiden Fällen das Pseudo-R ${ }^{2}$. Die zusätzliche

Tab. 3 Logistische Regression für die Wahrscheinlichkeit einer Änderung in der Unternehmensstruktur und im Führungsstil

\begin{tabular}{|c|c|c|c|c|}
\hline \multirow[t]{2}{*}{ Variablen } & Modell 1 & Modell 2 & Modell 3 & Modell 4 \\
\hline & \multicolumn{2}{|c|}{$\begin{array}{l}\text { Änderung in der Organisationsstruk- } \\
\text { tur }\end{array}$} & \multicolumn{2}{|c|}{ Änderung im Führungsstil } \\
\hline Acquirer & - & $\begin{array}{l}-0,29 * * * \\
(0,06)\end{array}$ & - & $\begin{array}{l}-0,12^{*} \\
(0,07)\end{array}$ \\
\hline Nicht fusionierende & - & $\begin{array}{l}-0,33 * * * \\
(0,09)\end{array}$ & - & $\begin{array}{l}-0,22^{* *} \\
(0,11)\end{array}$ \\
\hline Aspirationsniveau & $\begin{array}{l}-0,17 * * * \\
(0,03)\end{array}$ & $\begin{array}{l}-0,12 * * * \\
(0,03)\end{array}$ & $\begin{array}{l}-0,11 * * * \\
(0,03)\end{array}$ & $\begin{array}{l}-0,09 * * \\
(0,03)\end{array}$ \\
\hline Strategie-Struktur-Fit & $\begin{array}{l}-0,60 \\
(2,52)\end{array}$ & $\begin{array}{l}-0,33 \\
(2,35)\end{array}$ & - & - \\
\hline Strategie-Führungsstil-Fit & - & - & $\begin{array}{l}0,02 \\
(0,43)\end{array}$ & $\begin{array}{l}-0,04 \\
(0,44)\end{array}$ \\
\hline Organisationsstruktur & $\begin{array}{l}0,26 \\
(0,33)\end{array}$ & $\begin{array}{l}0,31 \\
(0,31)\end{array}$ & - & - \\
\hline Führungsstil & - & - & $\begin{array}{l}0,15^{* * *} \\
(0,05)\end{array}$ & $\begin{array}{l}0,16^{* * * *} \\
(0,05)\end{array}$ \\
\hline Größe & $\begin{array}{l}-0,12 * * * \\
(0,04)\end{array}$ & $\begin{array}{l}-0,10 * * * \\
(0,03)\end{array}$ & $\begin{array}{l}-0,09 * * * \\
(0,03)\end{array}$ & $\begin{array}{l}-0,08^{* *} \\
(0,04)\end{array}$ \\
\hline $\begin{array}{l}\text { Wahrgenommene Verände- } \\
\text { rung der Umwelt }\end{array}$ & $\begin{array}{l}-0,01 \\
(0,03)\end{array}$ & $\begin{array}{l}-0,01 \\
(0,03)\end{array}$ & $\begin{array}{l}0,01 \\
(0,03)\end{array}$ & $\begin{array}{l}0,00 \\
(0,03)\end{array}$ \\
\hline Vergangene Zeit & $\begin{array}{l}-0,05 \\
(0,14)\end{array}$ & $\begin{array}{l}-0,00 \\
(0,13)\end{array}$ & $\begin{array}{l}0,23 * \\
(0,14)\end{array}$ & $\begin{array}{l}025^{*} \\
(0,14)\end{array}$ \\
\hline Beobachtungen & 230 & 230 & 230 & 230 \\
\hline Jahr FE & JA & $\mathrm{JA}$ & JA & $\mathrm{JA}$ \\
\hline Land FE & $\mathrm{JA}$ & $\mathrm{JA}$ & $\mathrm{JA}$ & $\mathrm{JA}$ \\
\hline Branche FE & JA & JA & JA & JA \\
\hline InterviewerFE & $\mathrm{JA}$ & $\mathrm{JA}$ & $\mathrm{JA}$ & $\mathrm{JA}$ \\
\hline Log-Likelihood & $-120,7$ & $-108,6$ & $-119,1$ & $-116,6$ \\
\hline Chi2 & 71,79 & 95,97 & 78,13 & 83,18 \\
\hline$p$-Wert & 0,000525 & $1,05 \mathrm{e}-06$ & $9,09 \mathrm{e}-05$ & $4,38 \mathrm{e}-05$ \\
\hline Pseudo-R2 & 0,229 & 0,306 & 0,247 & 0,20 \\
\hline
\end{tabular}

Standardfehler in Klammern

$* * * p<0,01 ; * * p<0,05 ; * p<0,1$ 
Erklärungskraft für Änderungen der Organisationsstruktur ist jedoch höher als für Änderungen des Führungsstils.

Modell 1 aus Tab. 3 gibt das Basismodell für die Änderung der Organisationsstruktur an. Wir sehen, dass die Wahrscheinlichkeit, dass Unternehmen Änderungen an ihrer Organisationsstruktur vornehmen umso kleiner ist, je mehr Unternehmen ihre eigenen Ansprüche erfüllen und dass die Größe einen signifikant negativen Einfluss auf die Wahrscheinlichkeit einer Änderung in der Organisationsstruktur hat. Beide marginalen Effekte bleiben in Modell 2 signifikant, wenn die Dummies für Käufer und nicht fusionierende Unternehmen hinzugefügt werden. Die marginalen Effekte der Variablen für Käufer und nicht-fusionierende Unternehmen sind negativ und hochsignifikant $(p<0,01)$, was darauf hinweist, dass bei übernommenen Unternehmen die Wahrscheinlichkeit einer Änderung der Organisationsstruktur signifikant höher ist als bei den anderen Unternehmenstypen. Dieses Ergebnis stützt Hypothesen 1a und 2a, nach denen übernommene Unternehmen eine höhere Wahrscheinlichkeit für eine Änderung der Organisationsstruktur aufweisen als Käufer (1a) und Unternehmen, die nicht an M\&As teilgenommen haben (2a).

Die Regressionsergebnisse zur Änderung des Führungsstils sind in den Modellen 3 und 4 von Tab. 3 angegeben. Wieder zeigt Modell 3 das Basismodell an. Das Erfüllen der eigenen Aspirationen und eine zunehmende Größe reduzieren beide signifikant die Wahrscheinlichkeit für Änderungen der Organisationsstruktur. Für eine Änderung des Führungsstils scheint jedoch auch der „Initialzustand“ von Bedeutung zu sein, jedoch anders als erwartet: Je „extremer“ Unternehmen anfangs in Bezug auf ihren Führungsstil waren, desto höher ist die Wahrscheinlichkeit einer Änderung des Führungsstils. Dieses Ergebnis ist hochsignifikant $(p<0,01)$ und bleibt auch nach dem Hinzufügen von Dummies für den Unternehmenstyp hochsignifikant. Auch die Zeit scheint für eine Änderung des Führungsstils eine Rolle zu spielen: In Modell 3 und 4 hat die vergangene Zeit einen signifikant positiven Einfluss auf die Wahrscheinlichkeit einer Änderung des Führungsstils, was darauf hindeutet, dass die Anpassung der Führungsstile möglicherweise einige Zeit in Anspruch nimmt.

Die in Modell 4 hinzugefügten marginalen Effekte für Käufer und nicht-fusionierende Unternehmen sind negativ und signifikant ( $p<0,1$ für Käufer und $p<0,05$ für nicht-fusionierende Unternehmen). Somit werden die Hypothesen $1 \mathrm{~b}$ und $2 \mathrm{~b}$ gestützt, die besagen, dass übernommene Unternehmen eine höhere Wahrscheinlichkeit haben, den Führungsstil zu ändern als Käufer (1b) und nicht fusionierende Unternehmen (2b).

Die Regressionen zum Testen der Hypothesen 3 und 4 sind in Tab. 4 gezeigt. Hypothese 3 sagt voraus, dass übernommene Unternehmen eher ihre Organisationsstruktur ändern als ihren Führungsstil. Um dies zu testen, haben wir die Modelle 2 und 4 aus Tab. 3 neu geschätzt, wobei wir statt Dummies für Käufer und für nicht fusionierende Unternehmen einen Dummy für übernommene Unternehmen miteinbezogen. Die Ergebnisse werden in den Modellen 1 und 2 von Tab. 4 gezeigt. Sie spiegeln im Wesentlichen die Ergebnisse der Modelle 2 und 4 von Tab. 3 wider, wobei der Dummy für Targets einen statistisch signifikanten $(p<0,01$ für die Organisationsstruktur und $p<0,05$ für den Führungsstil) positiven marginalen Effekt auf Änderungen der Organisationsstruktur und des Führungsstils zeigt. 
Tab. 4 Logistische Regressionen für den Mediationseffekt einer Änderung in der Unternehmensstruktur auf den Einfluss einer Übernahme auf das übernommene Unternehmen auf eine Änderung des Führungsstils

\begin{tabular}{|c|c|c|c|}
\hline Variablen & $\begin{array}{l}\text { Modell } 1 \\
\text { Änderung in der Organisa- } \\
\text { tionsstruktur }\end{array}$ & $\begin{array}{l}\text { Modell } 2 \\
\text { Änderung im } \\
\text { Führungsstil }\end{array}$ & $\begin{array}{l}\text { Modell } 3 \\
\text { Änderung im } \\
\text { Führungsstil }\end{array}$ \\
\hline Target & $\begin{array}{l}0,34 * * * \\
(0,07)\end{array}$ & $\begin{array}{l}0,14 * * \\
(0,07)\end{array}$ & $\begin{array}{l}0,07 \\
(0,07)\end{array}$ \\
\hline $\begin{array}{l}\text { Änderung in der Organisa- } \\
\text { tionsstruktur }\end{array}$ & - & - & $\begin{array}{l}021 * * * \\
(0,07)\end{array}$ \\
\hline Aspirationsniveau & $\begin{array}{l}-0,12^{* * * *} \\
(0,03)\end{array}$ & $\begin{array}{l}-0,03 * * \\
(0,04)\end{array}$ & $\begin{array}{l}-0,06 * \\
(0,04)\end{array}$ \\
\hline Strategie-Struktur-Fit & $\begin{array}{l}-0,32 \\
(2,34)\end{array}$ & - & - \\
\hline Strategie-Führungsstil-Fit & - & $\begin{array}{l}-0,03 \\
(0,44)\end{array}$ & $\begin{array}{l}0,01 \\
(0,43)\end{array}$ \\
\hline Organisationsstruktur & $\begin{array}{l}0,32 \\
(0,31)\end{array}$ & - & - \\
\hline Führungsstil & - & $\begin{array}{l}0,16 * * * \\
(0,05)\end{array}$ & $\begin{array}{l}0,16 * * \\
(0,05)\end{array}$ \\
\hline Größe & $\begin{array}{l}-0,09 * * * \\
(0,03)\end{array}$ & $\begin{array}{l}-0,03 * * \\
(0,03)\end{array}$ & $\begin{array}{l}-0,05 \\
(0,03)\end{array}$ \\
\hline $\begin{array}{l}\text { Wahrgenommene Verände- } \\
\text { rung der Umwelt }\end{array}$ & $\begin{array}{l}-0,01 \\
(0,03)\end{array}$ & $\begin{array}{l}0,00 \\
(003)\end{array}$ & $\begin{array}{l}0,01 \\
(0,03)\end{array}$ \\
\hline Vergangene Zeit & $\begin{array}{l}-0,00 \\
(0,13)\end{array}$ & $\begin{array}{l}0,26 * \\
(0,14)\end{array}$ & $\begin{array}{l}0,29 * * \\
(0,14)\end{array}$ \\
\hline Beobachtungen & 230 & 230 & 230 \\
\hline Jahr FE & JA & JA & JA \\
\hline Land FE & JA & JA & JA \\
\hline Branche FE & JA & JA & JA \\
\hline Interviewer FE & JA & JA & JA \\
\hline Log-Likelihood & $-108,8$ & $-116,9$ & $-112,2$ \\
\hline Chi2 & 95,62 & 82,45 & 91,98 \\
\hline$p$-Wert & $721 \mathrm{e}-07$ & $398 \mathrm{e}-05$ & $3,6 \mathrm{Ce}-06$ \\
\hline Pseudo-R2 & 0,305 & 0,261 & 0,291 \\
\hline
\end{tabular}

Standardfehler in Klammern

$* * * p<0,01 ; * * p<0,05 ; * p<0,1$

Um Hypothese 3 zu untersuchen, haben wir die marginalen Effekte für den Dummy der übernommene Unternehmen für beide abhängigen Variablen in jeder einzelnen Beobachtung berechnet (Cameron und Trivedi 2009). In einem zweiten Schritt haben wir die Mittelwerte der beiden durchschnittlichen marginalen Effekte (auf Führungsstil und Organisationsstruktur) mit einem gepaarten t-Test verglichen. Es gibt einen hochsignifikanten $(p<0,01)$ Unterschied zwischen den beiden marginalen Effekten. Zudem ist der marginale Effekt des Dummys für übernommene Unternehmen bezüglich einer Änderung der Organisationsstruktur um etwa 0,2 größer als der marginale Effekt auf eine Änderung des Führungsstils. Dies bestätigt Hypothese 3. 
Tab. 5 OLS-Regressionen für das Ausmaß der Änderung in der Unternehmensstruktur und im Führungsstil

\begin{tabular}{|c|c|c|c|c|c|}
\hline Variablen & $\begin{array}{l}\text { Modell } 1 \\
\text { Änderung in } \\
\text { der Organisati- } \\
\text { onsstruktur } \\
\text { (Ausmaß) }\end{array}$ & $\begin{array}{l}\text { Modell } 2 \\
\text { Änderung } \\
\text { im Füh- } \\
\text { rungsstil } \\
\text { (Ausmaß) }\end{array}$ & $\begin{array}{l}\text { Modell } 3 \\
\text { Änderung in } \\
\text { der Organisati- } \\
\text { onsstruktur } \\
\text { (Ausmaß) }\end{array}$ & $\begin{array}{l}\text { Modell } 4 \\
\text { Änderung } \\
\text { im Füh- } \\
\text { rungsstil } \\
\text { (Ausmaß) }\end{array}$ & $\begin{array}{l}\text { Modell } 5 \\
\text { Änderung } \\
\text { im Füh- } \\
\text { rungsstil } \\
\text { (Ausmaß) }\end{array}$ \\
\hline Acquirer & $\begin{array}{l}-0,35 * * \\
(0,08)^{*}\end{array}$ & $\begin{array}{l}-0,83^{* * *} \\
(0,41)\end{array}$ & - & - & - \\
\hline $\begin{array}{l}\text { Nicht fusionie- } \\
\text { rende }\end{array}$ & $\begin{array}{l}-0,49 * * * \\
(0,14)\end{array}$ & $\begin{array}{l}-1,31 * \\
(0,70)\end{array}$ & - & - & - \\
\hline Target & - & - & $\begin{array}{l}0,37 * * * \\
(0,08)\end{array}$ & $\begin{array}{l}0,92 * * * \\
(0,38)\end{array}$ & $\begin{array}{l}0,43 \\
(0,39)\end{array}$ \\
\hline $\begin{array}{l}\text { Veränderung } \\
\text { in der Organi- } \\
\text { sationsstruktur } \\
\text { (Ausmaß) }\end{array}$ & - & - & - & - & $\begin{array}{l}1,31 * * * \\
(0,35)\end{array}$ \\
\hline Aspirationsniveau & $\begin{array}{l}-0,03 \\
(0,04)\end{array}$ & $\begin{array}{l}-0,69 * * * \\
(0,21)\end{array}$ & $\begin{array}{l}-0,02 \\
(0,04)\end{array}$ & $\begin{array}{l}-0,67 * * * \\
(0,21)\end{array}$ & $\begin{array}{l}-0,66^{* * * *} \\
(0,20)\end{array}$ \\
\hline $\begin{array}{l}\text { Strategie-Struk- } \\
\text { tur-Fit }\end{array}$ & $\begin{array}{l}0,39 \\
(2,98)\end{array}$ & - & 0,45 & - & - \\
\hline $\begin{array}{l}\text { Strategie-Füh- } \\
\text { rungsstil-Fit }\end{array}$ & - & $\begin{array}{l}0,96 \\
(2,25)\end{array}$ & - & $\begin{array}{l}1,07 \\
(2,24)\end{array}$ & $\begin{array}{l}1,52 \\
(2,17)\end{array}$ \\
\hline $\begin{array}{l}\text { Organisations- } \\
\text { struktur }\end{array}$ & $\begin{array}{l}0,64 \\
(0,39)\end{array}$ & - & $\begin{array}{l}0,64 * \\
(0,39)\end{array}$ & - & - \\
\hline Führungsstil & - & $\begin{array}{l}2,91 * * * \\
(0,31)\end{array}$ & - & $\begin{array}{l}2,93 * * * \\
(0,31)\end{array}$ & $\begin{array}{l}2,80 * * * \\
(0,30)\end{array}$ \\
\hline Größe & $\begin{array}{l}-0,15^{* * *} \\
(0,05)\end{array}$ & $\begin{array}{l}-0,21 \\
(0,21)\end{array}$ & $\begin{array}{l}-0,14 * * * \\
(0,05)\end{array}$ & $\begin{array}{l}-0,17 \\
(0,20)\end{array}$ & $\begin{array}{l}0,02 \\
(0,20)\end{array}$ \\
\hline $\begin{array}{l}\text { Wahrgenommene } \\
\text { Veränderung der } \\
\text { Umwelt }\end{array}$ & $\begin{array}{l}-0,02 \\
(0,03)\end{array}$ & $\begin{array}{l}0,05 \\
(0,16)\end{array}$ & $\begin{array}{l}-0,02 \\
(0,03)\end{array}$ & $\begin{array}{l}0,05 \\
(0,16)\end{array}$ & $\begin{array}{l}0,07 \\
(0,15)\end{array}$ \\
\hline Vergangene Zeit & $\begin{array}{l}0,04 \\
(0,07)\end{array}$ & $\begin{array}{l}0,41 \\
(0,35)\end{array}$ & $\begin{array}{l}0,01 \\
(0,06)\end{array}$ & $\begin{array}{l}0,30 \\
(0,32)\end{array}$ & $\begin{array}{l}0,28 \\
(0,30)\end{array}$ \\
\hline $\begin{array}{l}\text { Vorige Anzahl } \\
\text { von Hierarchie- } \\
\text { ebenen }\end{array}$ & $\begin{array}{l}0,00 \\
(0,04)\end{array}$ & - & $\begin{array}{l}0,01 \\
(0,04)\end{array}$ & - & - \\
\hline Konstante & $\begin{array}{l}1,50 * * * \\
(0,54)\end{array}$ & $\begin{array}{l}8,45^{* * *} \\
(2,67)\end{array}$ & $\begin{array}{l}1,16^{* *} \\
(0,56)\end{array}$ & $\begin{array}{l}7,62 * * * \\
(2,75)\end{array}$ & $\begin{array}{l}6,16 * * \\
(2,68)\end{array}$ \\
\hline Beobachtungen & 230 & 230 & 230 & 230 & 230 \\
\hline Jahr FE & JA & JA & JA & JA & JA \\
\hline Land FE & JA & JA & JA & JA & JA \\
\hline Branche FE & JA & JA & JA & JA & JA \\
\hline Interviewer FE & JA & JA & JA & JA & JA \\
\hline$p$-Wert & $8,76 \mathrm{e}-05$ & 0 & $7,43 e-05$ & 0 & 0 \\
\hline $\mathrm{R} 2$ & 0,329 & 0,523 & 0,325 & 0,521 & 0,555 \\
\hline
\end{tabular}

Standardfehle $\mathrm{r}$ in Klammern

$* * * p<0,01 ; * * p<0,05 ; * p<0,1$ 
Wir folgen dem Ansatz von Baron und Kenny (1986), um zu testen, ob Änderungen des Führungsstils in Zielunternehmen durch Veränderungen in der Organisationsstruktur mediiert werden (Batt 2002; Baum und Wally 2003; Oyewobi et al. 2016; Collins und Clark 2003; Mithas et al. 2005; Yli-Renko et al. 2001). Folgende Bedingungen müssen erfüllt sein, um einen Mediationseffekt zu identifizieren:

1. Es muss einen signifikanten Einfluss der unabhängigen Variable (übernommenes Unternehmen) auf den Mediator geben (Änderung der Organisationsstruktur).

2. Die unabhängige Variable (übernommenes Unternehmen) muss bei Abwesenheit des Mediators einen signifikanten Einfluss auf die abhängige Variable haben ( $̈$ nderung des Führungsstils).

3. Es muss einen signifikanten Effekt der Mediatorvariablen auf die abhängige Variable geben, wenn diese Variable in Gegenwart der unabhängigen Variablen hinzugefügt wird; und schließlich

4. muss sich der Einfluss der unabhängigen Variable auf die abhängige Variable in Gegenwart der mediierenden Variablen verringern.

Tab. 4 lässt uns die verschiedenen Bedingungen identifizieren. Der Dummy für Zielunternehmen hat einen signifikanten Effekt auf die Änderung der Organisationsstruktur und des Führungsstils, wie in den Modellen 1 und 2 von Tab. 4 zu sehen ist. Wir haben die Änderung der Organisationsstruktur zur Regression der Änderung des Führungsstils in Modell 3 hinzugefügt. Hier hat eine Änderung der Organisationsstruktur einen hochsignifikanten positiven Einfluss auf die Änderung des Führungsstils $(p<0,01)$. Beim Vergleich der Modelle 2 und 3 wird der marginale Effekt des Dummys für übernommene Unternehmen in Modell 3 (einschließlich der Änderung der Organisationsstruktur) insignifikant und ist in Modell 3 geringer als in Modell 2 (ohne Dummy). Dies deutet darauf hin, dass Veränderungen im Führungsstil in akquirierten Unternehmen vollständig durch eine Veränderung der Organisationsstruktur mediiert werden, was Hypothese 4 stützt.

In einem Robustheitstest haben wir als abhängige Variable nicht die Wahrscheinlichkeit einer Änderung (der Organisationsstruktur bzw. des Führungsstils), sondern das tatsächliche Ausmaß der Änderung in einer linearen Regression verwendet. ${ }^{10}$ Diese beiden abhängigen Variablen erfassen die absolute Anzahl von Veränderungen, die in Bezug auf alle sechs Entscheidungsrechte vorgenommen wurden (Organisationsstruktur) bzw. die absolute Anzahl von Veränderungen, die bei den fünf Items zum Partizipationsgrad erfolgt sind (Führungsstil). Die Ergebnisse sind in Tab. 5 und entsprechen den Ergebnissen der Logit-Modelle.

In den Modellen 1 und 2 von Tab. 5 sehen wir, dass übernommene Unternehmen ein signifikant höheres Ausmaß an Änderungen in der Organisationsstruktur und im Führungsstil aufweisen als Käufer und nicht-fusionierende Unternehmen. In beiden Modellen sind Koeffizienten für Käufer und nicht-fusionierende Unternehmen negativ und signifikant. Um die Hypothesen 3 und 4 zu untersuchen, wurden in Tab. 5 die Modelle 3-5 geschätzt. Entsprechend dem bisherigen Vorgehen wurden die Dum-

\footnotetext{
10 Wir haben eine Anpassung in den OLS-Regressionen zur Organisationsstruktur vorgenommen. Aus technischen Gründen haben wir die Anzahl der bis zur Akquisition vorhandenen Hierarchieebenen als zusätzliche Kontrollvariable in die Regressionen aufgenommen.
} 
mies für Käufer und für nicht-fusionierende Unternehmen durch einen Dummy für übernommene Unternehmen ersetzt. Ein Vergleich der Koeffizienten zeigt wiederum, dass Hypothese 3 gestützt wird: Zielunternehmen weisen etwa um 0,37 höhere Veränderungen des Dezentralisierungsgrades von Entscheidungsrechten auf als die anderen Unternehmen, was etwa $70 \%$ der Standardabweichung der abhängigen Variablen entspricht $(\mathrm{sd}=0,53)$. In Bezug auf den Führungsstil weisen Zielunternehmen im Vergleich zu nicht-fusionierenden Unternehmen und Käufern einen größeren absoluten Wert der Veränderung von etwa 0,92 auf. Dies entspricht jedoch nur etwa $30 \%$ der Standardabweichung der abhängigen Variablen, dem Ausmaß der Änderung des Führungsstils ( $\mathrm{sd}=3,11)$. Insgesamt deuten unsere OLS-Ergebnisse erneut darauf hin, dass übernommene Unternehmen ihre Organisationsstruktur mit höherer Wahrscheinlichkeit ändern als den Führungsstil. Schließlich sind, wie in den Modellen 3-5 gezeigt wird, erneut alle von Baron und Kenny (1986) bereitgestellten Kriterien zur Identifizierung eines Mediationseffektes erfüllt.

\subsection{Alternative Erklärungen}

Wir diskutieren nun einige alternative Erklärungen für unsere Ergebnisse.

1. Zunächst kann es sein, dass die höheren Veränderungswahrscheinlichkeiten in den übernommenen Unternehmen nicht durch die Akquisition verursacht wurden, sondern dass diese (oder die zugrundeliegenden Ursachen dafür) sie erst anfällig für Akquisitionen machen. Das scheint aus zwei Gründen nicht plausibel: Zum einen bezogen sich unsere Fragen spezifisch auf die Zeitfenster vor und nach der Transaktion. Änderungen ergeben sich somit aus Unterschieden zwischen der Ex-Ante- und der Ex-Post-Situation. Dies bedeutet, dass wir die folgende logische Kette von Aktivitäten messen: anfängliche Organisationsstruktur/Führungsstil $\rightarrow$ M\&A $\rightarrow$ Ex-Post (angepasste) Organisationsstruktur/ Führungsstil. Die Logik der gestellten Fragen impliziert also, dass die Änderung chronologisch nach der Transaktion stattgefunden hat. Zum anderen hinaus ist es unwahrscheinlich, dass eine (anstehende) Änderung der Organisationsstruktur oder des Führungsstils allein dazu führen würde, dass ein Unternehmen gekauft wird. Vielmehr ist es naheliegend, dass es Ereignisse gibt, die beides kausal verursachen. Intuitiv wäre zum Beispiel ungenügender Unternehmenserfolg, die Manager dazu veranlasst, Änderungen in Betracht zu ziehen und gleichzeitig die Verkaufsbereitschaft der Eigentümer zu erhöhen. Dieser Effekt sollte jedoch zumindest teilweise von der Variable „Aspirationsniveau“ erfasst werden.

2. Ebenso kann die Anstellung eines neuen Managers Änderungen (in Führungsstil und Organisationsstruktur) im Target auslösen und das Unternehmen für potenzielle Bieter attraktiver machen. Auch hier sollte allerdings intuitiv ein neuer Manager die Attraktivität für externe Bieter nicht erhöhen (wenn überhaupt, könnte ein längerfristiger Vertrag die Entlassungskosten erhöhen), zumal Manager das Zielunternehmen häufig kurz nach der Akquisition verlassen.

3. Schließlich können inhärente Unterschiede zwischen Zielunternehmen und anderen Unternehmenstypen in Bezug auf Führungsstile oder Organisationsstrukturen unser Ergebnis beeinflussen. Dies würde bedeuten, dass der Ausgangszustand der 
Organisationsstruktur oder des Führungsstils die Wahrscheinlichkeit einer Änderung beeinflusst, ebenso wie die Tatsache, dass das Unternehmen akquiriert wurde. Wie bereits erwähnt erscheint es nicht plausibel, dass eine bestimmte Art von Führungsstil oder Organisationsstruktur per se die Akquisition eines Unternehmens auslöst. Wieder ist es vielmehr der resultierende Unternehmenserfolg (oder potenzielle Unternehmenserfolg), die z.B. durch die Fähigkeit entsteht, eine Strategie effizient zu implementieren, die ein Unternehmen attraktiv macht. Zusätzlich zu der Variable „Aspirationsniveau“, die die aktuelle Performance erfasst, wird dies auch in den Strukturvariablen zum Fit erfasst.

\section{Diskussion und Fazit}

\subsection{Zentrale Erkenntnisse und Forschungsbeiträge}

Wir analysieren Veränderungen in der Organisationsstruktur und im Führungsstil in akquirierten Unternehmen. Bestehende Studien analysierten weitgehend die Integrationsbemühungen und sahen die innerhalb der übernommenen Unternehmen eingeleiteten Veränderungen als gegeben an. Diese weitgehend anekdotischen Belege für Veränderungsprozesse in Targets haben wir in der vorliegenden Studie durch eine groß angelegte empirische Analyse untermauert und weitergehend untersucht.

Unsere Ergebnisse stützen die weit verbreitete Überzeugung, dass Zielunternehmen tendenziell einer verstärkten Veränderungsaktivität unterliegen. Sie haben eine signifikant höhere Wahrscheinlichkeit dafür, sich sowohl in Bezug auf die Organisationsstruktur als auch in Bezug auf den Führungsstil zu ändern, im Gegenzug zu Käufern und Unternehmen, die an keiner M\&A-Transaktion beteiligt waren. Dies könnte auch einige der Gründe für den oftmals enttäuschenden Erfolg von Fusionen und Akquisitionen aufzeigen: Die Zielunternehmen verändern sich überproportional, was (unerwartete) Anpassungskosten verursachen kann. Darüber hinaus hat die Akquisition einen höheren Einfluss auf eine Änderung der Organisationsstruktur als auf eine Änderung des Führungsstils, wobei Veränderungen in beiden Aspekten zusammenhängen: Eine Änderung der Organisationsstruktur erleichtert eine Änderung des Führungsstils in Zielunternehmen. Insgesamt stützen unsere Ergebnisse die anekdotischen Belege für Veränderungen in übernommenen Unternehmen (Schweiger und Csiszar 2002; Schweizer 2005).

Wir tragen auf vielfältige Weise zur vorhandenen Literatur bei. Erstens schließen wir eine wichtige konzeptionelle Lücke zwischen M\&As und Änderungen im Organisationsdesign von Zielunternehmen. Es wird oft angenommen, dass Integrationsprozesse nach Übernahmen substantielle Veränderungen in den Zielunternehmen nach sich ziehen, die wiederum Unsicherheit unter den Angestellten schürt (Cartwright und Cooper 1990; Larsson und Finkelstein 1999). Wir bieten einen empirischen Beleg dafür, dass die übernommenen Unternehmen tatsächlich einer signifikant erhöhten Wahrscheinlichkeit für Veränderung in der Organisationsstruktur und im Führungsstil ausgesetzt sind. Daher ist das Phänomen, dass sich Käufer auf Anpassungen in den übernommenen Unternehmen konzentrieren, die folgerichtig zu verstärkten Veränderungsaktivitäten in diesen Organisationseinheiten führen, mehr 
als nur anekdotisch. Gleichzeitig ruft dieses Ergebnis angesichts der potenziell negativen Effekte von Änderungen im Organisationsdesign auf den Unternehmenserfolg zu mehr Forschung zu diesem Thema auf (Lamont et al. 1994; Oxman und Smith 2003).

Darüber hinaus erweitern wir den Forschungsumfang zu Post-Merger-Integrationsprozessen, indem wir über die Beziehung zwischen Käufer und gekauftem Unternehmen hinausgehen und diese mit einer ,nicht betroffenen“ Unternehmensgruppe vergleichen. Dies ist aus mindestens zwei Gründen nützlich: Zum einen wird die Post-Merger-Performance häufig mit nicht fusionierenden Unternehmen verglichen - beispielsweise anhand der Aktienrendite. Wenn also die Kosten für die Integrationsprozesse nach Akquisitionen dafür verantwortlich sind, dass M\&As nicht zu einer überdurchschnittlichen Aktienrendite führen, ist es zielführend, nichtfusionierende Unternehmen mit Zielunternehmen und Käufern zu vergleichen, um den oft vergleichsweise geringen Erfolg des fusionierten Unternehmens zu erklären. Zum anderen ist es interessant zu analysieren, inwieweit M\&As ,unerwartete“ Veränderungsprozesse auslösen, die nicht nur bereits stattfindende reguläre Veränderungen beschleunigen. Verschiedene Autoren haben die permanenten Wechsel zwischen Zentralisierung und Dezentralisierung hervorgehoben, die regelmäßig in Unternehmen stattfinden (Raveendran 2020) und die Frage aufgeworfen, inwieweit M\&As die Wahrscheinlichkeit einer Änderung der Organisationsstruktur in Zielunternehmen weiter erhöhen (Cummings 1995; Mintzberg 1979).

Wir tragen auch zur Forschung zu Veränderungsprozessen in übernommenen Unternehmen bei. Während sich Zielunternehmen bezüglich der Wahrscheinlichkeit von Änderungen sowohl in der Organisationsstruktur als auch im Führungsstil von Käufern und nicht fusionierenden Unternehmen unterscheiden, ist dieser Unterschied im Hinblick auf die Organisationsstruktur signifikant größer. Dies kann zunächst auf die Tatsache zurückzuführen sein, dass die Änderung des Führungsstils relativ zeitaufwendig ist. Dies sollte zu einem einfachen Zeiteffekt führen, der durch die Signifikanz der Variable ,vergangene Zeit“" in unseren Ergebnissen angezeigt wird. Jedoch gibt es auch plausible alternative Erklärungen: Beispielsweise können sich Käufer darauf konzentrieren, die formale Struktur so auszurichten, dass Synergien koordiniert und realisiert werden, wobei der informellen Organisation weniger Aufmerksamkeit geschenkt wird (Gulati und Puranam 2009). Dies würde vorübergehende Inkonsistenzen zwischen der formellen und der informellen Organisation schaffen und signifikante Leistungseinbußen riskieren (Lamont et al. 1994).

Darüber hinaus weisen unsere Ergebnisse auf einen Zusammenhang zwischen einer Änderung der Organisationsstruktur der Zielunternehmen und einer Änderung ihres Führungsstils hin, wobei erstere letztere fördert. Dies steht im Einklang mit Schweizer (2005), der feststellt, dass Änderungen der Organisationsstrukturen zu Änderungen der Organisationskultur führten. Käufer, die durch die Umsetzung struktureller Veränderungen in den Zielunternehmen die Effizienz steigern möchten, laufen daher Gefahr, den für das Unternehmen wichtigen und idiosynkratischen Führungsstil zu beeinträchtigen. Folglich können diese Änderungen zusätzlich zum „direkten“ negativen Einfluss auf den Unternehmenserfolg, der sich durch Personalprobleme bei der Änderung der Organisationsstruktur ergibt, auch ,indirekt“ den 
Unternehmenserfolg beeinträchtigen, indem sie unvorhergesehene negative Veränderungen im Führungsstil verursachen.

\subsection{Implikationen für die Praxis}

Die Ergebnisse zu den Hypothesen 3 und 4 haben besondere praktische Relevanz. Einerseits sollten Manager erkennen, dass Veränderungen der Organisationsstruktur des Zielunternehmens zwar relativ einfach zu implementieren sind, aber gleichermaßen anspruchsvollere Veränderungen des Führungsstils angestrebt werden sollten, um Inkonsistenzen zwischen der formellen und der informellen Organisation im Zielunternehmen zu vermeiden.

Allerdings sollten Manager auf mögliche Wechselwirkungen der strukturellen Veränderungen auf Änderungen im Führungsstil achten, wenn sie strukturelle Veränderungen implementieren, um Synergien zu erzielen und den potenziell gewinnbringenden idiosynkratischen Führungsstil des übernommenen Unternehmens dabei beibehalten. Durch die Anpassung der Organisationsstruktur erhöhen Käufer die Wahrscheinlichkeit dafür, dass sich der Führungsstil in Zielunternehmen ändert, wodurch möglicherweise eine wertvolle Unternehmensressource verlorengeht. Um das zu vermeiden, könnten Manager entweder statt der strukturellen Ausrichtung alternativen Koordinierungsmechanismen mehr Aufmerksamkeit schenken oder versuchen, Gegenmaßnahmen zu ergreifen, um den unternehmensspezifischen Führungsstil beizubehalten.

\subsection{Fazit und Ausblick}

In unserer empirischen Analyse haben wir uns auf Produktionsunternehmen konzentriert. Das hat den Vorteil, dass physischen Vermögenswerten eine relativ hohe Bedeutung zukommt, wodurch es relativ unwahrscheinlich wird, dass gekaufte Produktionsstätten vollständig mit denen des Käufers zusammengelegt werden. Das führt zu einem relativ trennscharfen Vergleich der Vor- und Nachakquisitionsphase. Jedoch kann die Abhängigkeit von physischen Vermögenswerten auch die Wahrscheinlichkeit einer Änderung des Organisationsdesigns reduzieren, da bestehende Prozesse und Routinen zumindest teilweise von Produktionseinrichtungen determiniert werden können. Daher könnte es interessant sein, unsere Hypothesen in Dienstleistungsunternehmen zu testen.

Es ist anzumerken, dass unsere Datenbasis und die empirische Umsetzung keine eindeutige kausale Identifikation zulässt. Übernahmeziele werden nicht zufällig ausgewählt, und andere, parallele Prozesse können Veränderungen in Unternehmen anstoßen. Durch die Auswahl von „Unternehmenspaaren“ (so ist jedem übernommenen Unternehmen ein Käufer zugeordnet) haben wir versucht, derartige Störfaktoren weitestgehend auszuschließen, da diese Käufer und Zielunternehmen gleichermaßen von äußeren Einflüssen betroffen wären. Unbeobachtete Faktoren, die sowohl die Übernahmewahrscheinlichkeit als auch die Wahrscheinlichkeit strukturellen und kultureller Änderungen beeinflussen, können teilweise durch Verwendung von Kontrollvariablen erfasst werden, doch wäre ein natürliches Experiment, etwa in Form eines Vergleichs von erfolgreichen Übernahmen mit solchen, die aus wettbewerbs- 
rechtlichen Gründen verzögert oder geblockt wurden, noch besser geeignet, um eine klare Identifikation des Einflusses von Akquisitionen auf organisationale Veränderungsprozesse vorzunehmen.

Eine eingehendere Untersuchung, ob die vorhandenen Ergebnisse in verschiedenen Regionen und in ihrem spezifischen institutionellen Umfeld zutreffen, könnte weitere moderierende Faktoren aufdecken. Deutschland beispielsweise hat verhältnismäßig umfassenden Arbeitnehmerschutz (Capron und Guillén 2009). Folglich kann es für Käufer schwer sein, Ex-Post Änderungen zu implementieren und man würde ein insgesamt niedriges „Basisniveau“ von strukturellen Veränderungen erwarten. Eine empirische Studie in einem Setting mit weniger restriktiven arbeitsrechtlichen Bestimmungen könnte hier wichtige Erkenntnisse bieten.

Schließlich war die Zeitperiode der Umfrage auch durch makroökonomische Turbulenzen gekennzeichnet. Trotz der Tatsache, dass ein großer Teil der Beobachtungen aus deutschsprachigen Ländern stammt, die vergleichsweise wenig von diesen Turbulenzen betroffen waren, haben wir für das jeweilige Land der untersuchten Unternehmen kontrolliert. Die weniger stabile Wirtschaftslage in Spanien beeinflussen unsere Ergebnisse nicht maßgeblich.

Konzeptionell erfordert der Befund hinsichtlich des Mediationseffektes weitere Analysen zur Wechselwirkung zwischen verschiedenen Arten von Veränderungen nach einer Übernahme. Beispielsweise können Änderungen im Organisationsdesign auch die Strategie des Zielunternehmens beeinflussen, indem sie Prozesse des Wissensaustausches hemmen oder fördern. Bei Veränderungsprozessen nach Übernahmen sollten daher ganze Systeme zusammenhängender organisatorischer Merkmalen analysiert werden.

Unsere Ergebnisse werfen auch die normative Frage auf, wie Koordinierungsansätze entworfen werden müssen, um die erhofften positiven Effekte aus Unternehmensübernahmen zu realisieren. Puranam et al. (2009) zeigen hier bereits Ansätze auf, die den Wert von alternativen, weniger disruptiven Koordinationsmechanismen aufzeigen.

Abschließend hoffen wir, mit der vorliegenden Studie einen Beitrag zur Erklärung des Wechselspiels zwischen Akquisitionen, struktureller Transformation und Führungsstil geliefert zu haben und damit weitere Forschung zu diesem Thema anzustoßen.

Danksagung Wir bedanken uns bei den Mitarbeitern des Instituts für Strategie, Technologie und Organisation, insbesondere Leon Zucchini, Thorsten Grohsjean und Ferdinand Mahr für hilfreiche Kommentare, bei Isabell Schommers für die Übersetzung des Manuskripts sowie bei Caroline Heinemann für ihre wertvolle Unterstützung bei der finalen Überarbeitung des Manuskripts.

Förderung Diese Studie wurde mit finanzieller Unterstützung der Deutschen Forschungsgemeinschaft (Projektnummer KR 3479/5-1) durchgeführt.

Funding Open Access funding enabled and organized by Projekt DEAL.

Open Access Dieser Artikel wird unter der Creative Commons Namensnennung 4.0 International Lizenz veröffentlicht, welche die Nutzung, Vervielfältigung, Bearbeitung, Verbreitung und Wiedergabe in jeglichem Medium und Format erlaubt, sofern Sie den/die ursprünglichen Autor(en) und die Quelle ordnungsgemäß nennen, einen Link zur Creative Commons Lizenz beifügen und angeben, ob Änderungen vorgenommen wurden. 
Die in diesem Artikel enthaltenen Bilder und sonstiges Drittmaterial unterliegen ebenfalls der genannten Creative Commons Lizenz, sofern sich aus der Abbildungslegende nichts anderes ergibt. Sofern das betreffende Material nicht unter der genannten Creative Commons Lizenz steht und die betreffende Handlung nicht nach gesetzlichen Vorschriften erlaubt ist, ist für die oben aufgeführten Weiterverwendungen des Materials die Einwilligung des jeweiligen Rechteinhabers einzuholen.

Weitere Details zur Lizenz entnehmen Sie bitte der Lizenzinformation auf http://creativecommons.org/ licenses/by/4.0/deed.de.

Interessenkonflikt T. Kretschmer und C. Dehne geben an, dass kein Interessenkonflikt besteht.

\section{Anhang}

Übersicht über die Items, die zur Berechnung der Indizes für den Dezentralisierungsgrad von Entscheidungsrechten, den Partizipationsgrad, den Grad der relativen Exploration, die Integration von Aktivitäten, das Ausmaß des Ressourcentransfers, die Integrationsbemühungen und die Performance nach M\&As verwendet wurden

\section{Items zur Berechnung des Dezentralisierungsgrades von Entscheidungsrechten $(\alpha=0,79)$}

„Bitte geben Sie an, auf welcher Hierarchieebene folgende Entscheidungen in ihrem Unternehmen getroffen wurden:““

- Von den Produktionsmitarbeitern angewendete Arbeitsmethoden

- Zuweisung der Arbeit zu Produktionsmitarbeitern

- Lösen von Problemen im Produktionsprozess

- Entscheidung für oder gegen einen Bewerber für eine offene Stelle in der Herstellung

- Kündigung eines Mitarbeiters

- Festlegung von Richtlinien und Verfahrensanweisungen für Angestellte ohne Führungsverantwortung

- Einführung eines neuen Produktes

- Eintritt in einen neuen Markt

- Produktpreise

\section{Items zur Berechnung des Partizipationsgrades $(\alpha=0,84)$}

„Wie sehr stimmen Sie den folgenden Behauptungen zu?“

- Produktionsmitarbeiter ohne Führungsverantwortung wurden oft von Führungskräften darum gebeten, bei Entscheidungsprozessen mitzuwirken.

- Produktionsmitarbeiter ohne Führungsverantwortung war es erlaubt, Entscheidungen zu treffen.

- Produktionsmitarbeiter ohne Führungsverantwortung durften Verbesserungsvorschläge machen. 
Tab. 6 Faktoranalyse der Items zu Exploration und Exploitation

\begin{tabular}{llc}
\hline & Faktor 1 & Faktor 2 \\
\hline $\begin{array}{l}\text { Exploration }(\alpha=0,77) \\
\text { Herstellung von Produkten, die auf dem neuesten Stand der Technik }\end{array}$ & 0,316 & 0,556 \\
sind & & \\
Entwicklung neuer Produkte & 0,067 & 0,746 \\
Eröffnung neuer Technologiebereiche & 0,189 & 0,746 \\
Exploitation $(\alpha=0,77)$ & & \\
Kostenreduktion & 0,753 & 0,049 \\
Produktivitätssteigerungen & 0,817 & 0,193 \\
Strenge Qualitätskontrollen & 0,532 & 0,295 \\
\hline
\end{tabular}

(Skala: „1-stimme überhaupt nicht zu“ bis ,,5 - stimme voll und ganz zu“)

- Führungskräfte waren offen für Kritik durch Produktionsmitarbeiter ohne Führungsverantwortung.

- Führungskräfte bezogen die Meinungen, Ideen und Vorschläge der Produktionsmitarbeiter ohne Führungsverantwortung in den Entscheidungsprozess mit ein.

(Skala: „, - stimme überhaupt nicht zu“ bis ,,5 - stimme voll und ganz zu“).

\section{Items zur Berechnung der relativen Explorationsstärke (s. Tab. 6)}

„Wie sehr stimmen Sie dem zu, dass die folgenden strategischen Praktiken Kernkomponenten ihrer Unternehmensstrategie darstellen?“"

\section{Items zur Berechnung des Integrationsgrades von Aktivitäten $(\alpha=0,86)$}

„Wie hoch war der Grad der Post-Merger-Integration oder der Zusammenführung operativer Einheiten im Hinblick auf ..."

- Verwaltung

- Instandhaltung

- Einkauf

- Fertigungsprozess

- Lagerhaltung (Rohmaterialien und Endprodukte)

- Marketing

- Abgedeckte Produktmärkte

- Distributionskanäle

(Skala: „,1 - sehr niedrig“ bis ,,5- sehr hoch“).

V. Items zur Berechnung des Ausmaßes von Ressourcentransfers $(\alpha=0,82 / \alpha=0,83)$

„Bitte geben Sie das Ausmaß an, in dem ein Transfer von Fähigkeiten und KnowHow ihres Unternehmens auf das andere Unternehmen stattgefunden hat hinsichtlich ..." 
- Kapazitäten für Produktinnovationen

- Marketingexpertise

- Managementexpertise

- Know-How im Produktionsprozess

- Distributionsexpertise

- Lieferantenbeziehungen

(Skala: „1 - sehr niedrig“ bis ,5 - sehr hoch“).

\section{Items zur Berechnung der Integrationsbemühung $(\alpha=0,64)$}

„Bitte geben Sie an, auf welcher Hierarchieebene folgende Entscheidungen in ihrem Unternehmen getroffen wurden:“

„Bitte schätzen Sie den Grad des betrieblichen Zusammenwirkens zwischen den zusammengeschlossenen Unternehmen während der Integrationsperiode in Relation zur Gesamtheit der Aktivitäten in Ihrem Unternehmen (z. B. die Erzeugung tagtäglicher Materialien und Information, oder eine Restrukturierung, die aus einem permanenteren Austausch von Produkten, Einrichtungen, Personal oder anderer Ressourcen zwischen den Unternehmen resultiert)“

„Bitte schätzen Sie den Grad des koordinativen Aufwandes, der zur Förderung von Synergien angewandt wurde, indem das betriebliche Zusammenwirken zwischen den zusammengeschlossenen Unternehmen angepasst wurde. Dies kann abgeleitet werden vom Nutzungsgrad von Koordinationsmechanismen und -instrumenten der zusammengeschlossenen Unternehmen, wie spezielle Integratoren, Übergangsteams, Management-Infosysteme, Integrationspläne oder temporäre Austausche/Rotationen von Personal“.

(Skala: „1- sehr niedrig“ bis ,5-sehr hoch“).

VII. Items zur Berechnung der Post-M\&A-Performance $(\alpha=0,79)$

„Wie haben sich Marktanteil, Sales und die Rentabilität seit der Transaktion verändert?"“

- Marktanteil

- Sales

- Rentabilität in Relation zum Branchendurchschnitt

(Skala: „1 - starker Abfall“ bis „,5- starkes Wachstum“).

\section{Literatur}

Amburgey, T.L., D. Kelly, und W.P. Barnett. 1993. Resetting the clock: The dynamics of organizational change and failure. Administrative Science Quarterly 38(1):51-73.

Andersen, J.A., und P. Jonsson. 2006. Does organizational structure matter? On the relationship between the structure, functioning and effectiveness. International Journal of Innovation \& Technology Management 3(3):237-263.

Barkema, H.G., und M. Schijven. 2008. Toward unlocking the full potential of acquisitions: the role of organizational restructuring. The Academy of Management Journal 51(4):696-722. 
Baron, R.M., und D.A. Kenny. 1986. The moderator-mediator variable distinction in social psychological research: conceptual, strategic, and statistical considerations. Journal of Personality and Social Psychology 51(6):1173-1182.

Batt, R. 2002. Managing customer services: human resources practices, quit rates, and sales growth. Academy of Management Journal 45(3):587-597.

Bauer, F., et al, 2018. Examining links between pre and post M\&A value creation mechanisms-Exploitation, exploration and ambidexterity in central European SMEs. Long Range Planning 51(2):185-203.

Baum, R.J., und S. Wally. 2003. Strategic decision speed and firm performance. Strategic Management Journal 24(11):1107-1129.

Benner, M.J., und M.L. Tushman. 2003. Exploitation, exploration, and process management: the productivity dilemma revisited. The Academy of Management Review 28(2):238-256.

Björkman, I., G.K. Stahl, und E. Vaara. 2007. Cultural differences and capability transfer in cross-border acquisitions: The mediating roles of capability complementarity, absorptive capacity, and social integration. Journal of International Business Studies 38(4):658-672.

Bloom, N., R. Sadun, und J. van Reenen. 2008. Measuring and explaining decentralization across firms and countries. London: Centre for Economic Performance, London School of Economics and Political Science.

Buono, A.F., und J.L. Bowditch. 1989. The human side of mergers and acquisitions: managing collisions between people, cultures, and organization. San Francisco: Jossey-Bass.

Burton, R.M., J. Lauridsen, und B. Obel. 2002. Return on assets loss from situational and contingency misfits. Management Science 48(11):1461-1485.

Cameron, A.C., und P.K. Trivedi. 2009. Microeconometrics using stata. College Station: Stata Press.

Capron, L. 1999. The long-term performance of horizontal acquisitions. Strategic Management Journal 20(11):987-1018.

Capron, L., und M. Guillén. 2009. National corporate governance institutions and post-acquisition target reorganization. Strategic Management Journal 30(8):803-833.

Capron, L., und N. Pistre. 2002. When do acquirers earn abnormal returns? Strategic Management Journal 23(9):781-794.

Capron, L., P. Dussauge, und W. Mitchell. 1998. Resource redeployment following horizontal acquisitions in Europe and North America, 1988-1992. Strategic Management Journal 19(7):631-661.

Cartwright, S., und C.L. Cooper. 1990. The impact of mergers and acquisitions on people at work: existing research and issues. British Journal of Management 1(2):65-76.

Cartwright, S., und C.L. Cooper. 1993. The role of culture compatibility in successful organizational marriage. The Academy of Management Executive 7(2):47-70.

Cartwright, S., und R. Schoenberg. 2006. Thirty years of mergers and acqusitions research: recent advances and future opportunities. British Journal of Management 17(S1):1-5.

Ceci, F., F. Masciarelli, und A. Prencipe. 2016. Changes in organizational architecture: aspiration levels, performance gaps and organizational change. International Journal of Innovation and Technology Management 13(1):1650002.

Chandler, A.D., Jr.. 1962. Strategy and structure: chapters in the history of the American industrial enterprise. Cambridge: MIT Press.

Chang, S.-J., A. van Witteloostuijn, und L. Eden. 2010. From the Editors: common method variance in international business research. Journal of International Business Studies 41(2):178-184.

Chatterjee, S. 1992. Sources of value in takeovers: synergy or restructuring-implications for target and bidder firms. Strategic Management Journal 13(4):267-286.

Chatterjee, S., M.H. Lubatkin, D.M. Schweiger, und Y. Weber. 1992. Cultural differences and shareholder value in related mergers: linking equity and human capital. Strategic Management Journal 13(5):319-334.

Collins, C.J., und K.D. Clark. 2003. Strategic human resource practices, top management team social networks, and firm performance: the role of human resource practices in creating organizational competitive advantage. The Academy of Management Journal 46(6):740-751.

Coyle-Shapiro, J.A.-M. 1999. Employee participation and assessment of an organizational change intervention: a three wave study of Total Quality Management. The Journal of Applied Behavioural Science 35(4):439-456.

Cummings, S. 1995. Centralization and decentralization: the neverending story of separation and betrayal. Scandinavian Journal of Management 11(2):103-117.

Cyert, R.M., und J.G. March. 1963. A behavioral theory of the firm. Englewood Cliffs: Prentice Hall.

Dachler, H.P., und B. Wilpert. 1978. Conceptual dimensions and boundaries of participation in organizations: a critical evaluation. Administrative Science Quarterly 23(1):1-39. 
Datta, D.K. 1991. Organizational fit and acquisition performance: effects of post-acquisition integration. Strategic Management Journal 12(4):281-297.

Datta, D.K., und J.H. Grant. 1990. Relationships between type of acquisition, the autonomy given to the acquired firm, and acquisition success: an empirical analysis. Journal of Management 16(1):29-44.

Dehne, C. 2013. Essays on mergers and acquisitions-relatedness, strategy-structure fit, and organizational change. Dissertation. München: LMU.

Donaldson, L. 1987. Strategy and structural adjustment to regain fit and performance: in defence of contingency theory. Journal of Management Studies 24(1):1-24.

Gallie, D. 2013. Direct participation and the quality of work. Human Relations 66(4):453-473.

Graebner, M., et al, 2017. The process of postmerger integration: a review and agenda for future research. Academy of Management Annals 11(1):1-32.

Greve, H.R. 1998. Performance, aspirations, and risky organizational change. Administrative Science Quarterly 43(1):58-86.

Grohsjean, T., T. Kretschmer, und N. Stieglitz. 2015. Organizational resources and learning from ambiguous performance feedback. Munich: Mimeo, LMU.

Gulati, R., und P. Puranam. 2009. Renewal through reorganization: the value of inconsistencies between formal and informal organization. Organization Science 20(2):422-440.

Hambrick, D.C., und A.A. Cannella Jr.. 1993. Relative standing: a framework for understanding departures of acquired executives. The Academy of Management Journal 36(4):733-762.

Hanks, S.H., C.J. Watson, E. Jansen, und G.N. Chandler. 1993. Tightening the life-cycle construct: a taxonomic study of growth stage configurations in high-technology organizations. Entrepreneurship: Theory and Practice 18(2):5-29.

Hannan, M.T., und J. Freeman. 1984. Structural inertia and organizational change. American Sociological Review 49(2):149-164.

Harrison, J.S., M.A. Hitt, R.E. Hoskisson, und R.D. Ireland. 1991. Synergies and post-acquisition performance: differences versus similarities in resource allocations. Journal of Management 17(1):173-190.

Haspeslagh, P.C., und D.B. Jemison. 1991. Managing acquisitions: Creating value through corporate renewal. New York: The Free Press.

Heller, F.A. 1977. A longitudinal study in participative decision-making. Human Relations 30(7):567-587.

Hoetker, G. 2007. The use of logit and probit models in strategic management research: critical issues. Strategic Management Journal 28(4):331-343.

Homburg, C., und M. Bucerius. 2006. Is speed of integration really a success factor of mergers and acquisitions? An analysis of the role of internal and external relatedness. Strategic Management Journal 27(4):347-367.

Hunt, J.W. 1990. Changing pattern of acquisition behaviour in takeovers and the consequences for acquisition processes. Strategic Management Journal 11(1):69-77.

Kelly, D., und T.L. Amburgey. 1991. Organizational inertia and momentum: a dynamic model of strategic change. The Academy of Management Journal 34(3):591-612.

Kim, J.-Y., und S. Finkelstein. 2009. The effects of strategic and market complementarity on acquisition performance: evidence from the US commercial banking industry, 1989-2001. Strategic Management Journal 30(6):617-646.

King, D.R., D.R. Dalton, C.M. Daily, und J.G. Covin. 2004. Meta-analyses of post-acquisition performance: indications of unidentified moderators. Strategic Management Journal 25(2):187-200.

Krug, J.A., und W.H. Hegarty. 1997. Postacquisition turnover among U.S. top management teams: an analysis of the effects of foreign vs. domestic acquisitions of U.S. targets. Strategic Management Journal 18(8):667-675.

Labianca, G., J.F. Fairbank, G. Andrevski, und M. Parzen. 2009. Striving toward the future: aspiration-performance discrepancies and planned organizational change. Strategic Organization 7(4):433-466.

Lamont, B.T., R.J. Williams, und J.J. Hoffman. 1994. Performance during "M-form" reorganization and recovery time: the effects of prior strategy and implementation speed. The Academy of Management Journal 37(1):153-166.

Larsson, R., und S. Finkelstein. 1999. Integrating strategic, organizational, and human resource perspectives on mergers and acquisitions: a case survey of synergy realization. Organization Science 10(1):1-26.

Larsson, R., und M.H. Lubatkin. 2001. Achieving acculturation in mergers and acquisitions: an international case survey. Human Relations 54(12):1573-1607. 
Lee, C.C., und V. Grover. 2000. Exploring mediation between environmental and structural attributes: the penetration of communication technologies in manufacturing organizations. Journal of Management Information Systems 16(3):187-217.

Levinthal, D.A., und J.G. March. 1993. The myopia of learning. Strategic Management Journal 14(Special Issue: Organizations, Decision Making and Strategy):95-112.

Li, K., B. Qiu, und R. Shen. 2018. Organization capital and mergers and acquisitions. Journal of Financial and Quantitative Analysis 53(4):1871-1909.

Long, R.J. 1981. The effects of formal employee participation in ownership and decision making on perceived and desired patterns of organizational influence: a longitudinal study. Human Relations 34(10):847-876.

Lorsch, J.W. 1986. Managing culture: the invisble barrier to strategic change. California Management Review 28(2):95-109.

Lubatkin, M.H. 1987. Merger strategies and stockholder value. Strategic Management Journal 8(1):39-53.

Lubatkin, M., D. Schweiger, und Y. Weber. 1999. Top management turnover in related M\&A's: an additional test of the theory of relative standing. Journal of Management 25(1):55-73.

Mahr, F. 2010. Aligning information technology, organization, and strategy: effects on firm performance. Wiesbaden: Gabler.

March, J.G. 1991. Exploration and exploitation in organizational learning. Organization Science 2(1):71-87.

Miller, D. 1987. The structural and environmental correlates of business strategy. Strategic Management Journal 8(1):55-76.

Miller, D. 1992. Environmental fit versus internal fit. Organization Science 3(2):159-178.

Mintzberg, H. 1979. The structuring of organizations. Englewood Cliffs: Prentice-Hall.

Mithas, S., M.S. Krishnan, und C. Fornell. 2005. Why do customer relationship management applications affect customer satisfaction? Journal of Marketing 69(4):201-209.

Mood, C. 2010. Logistic regression: why we cannot do what we think we can do, and what we can do about it. European Sociological Review 26(1):67-82.

Nahavandi, A., und A.R. Malekzadeh. 1988. Acculturation in mergers and acquisitions. The Academy of Management Review 13(1):79-90.

Nahm, A.Y., M.A. Vonderembse, und X.A. Koufteros. 2003. The impact of organizational structure on time-based manufacturing and plant performance. Journal of Operations Management 21(3):281-306.

Nickerson, J.A., und T.R. Zenger. 2002. Being efficiently fickle: A dynamic theory of organizational choice. Organization Science 13(5):547-566.

Oxman, J.A., und B.D. Smith. 2003. The limits of structural change. MIT Sloan Management Review 45(1):77-82.

Oyewobi, L. O., Windapo, A. O., Rotimi, J. O. B. and Jimoh, R. A. 2016. Relationship between competitive strategy and construction organisation performance: The moderating role of organisational characteristics. Management Decision 54(9):2340-2366. https://doi.org/10.1108/MD-01-2016-0040.

Pablo, A.L. 1994. Determinants of acquisition integration level: a decision-making perspective. The Academy of Management Journal 37(4):803-836.

Phan, P.H., und C.W.L. Hill. 1995. Organizational restructuring and economic performance in leveraged buyouts: an ex post study. The Academy of Management Journal 38(3):704-739.

Podsakoff, P.M., S.B. MacKenzie, J.-Y. Lee, und N.P. Podsakoff. 2003. Common method biases in behavioral research: a critical review of the literature and recommended remedies. Journal of Applied Psychology 88(5):879-903.

Pugh, D.S. 1973. The measurement of organization structures. Organizational Dynamics 1(4):19-34.

Pugh, D.S., D.J. Hickson, C.R. Hinings, und C. Turner. 1968. Dimensions of organization structure. Administrative Science Quarterly 13(1):65-105.

Puranam, P., H. Singh, und S. Chaudhuri. 2009. Integrating acquired capabilities: when structural integration is (un)necessary. Organization Science 20(2):313-328.

Quinn, R.E., und K. Cameron. 1983. Organizational life cycles and shifting criteria of effectiveness: some preliminary evidence. Management Science 29(1):33-51.

Ramaswamy, K. 1997. The performance impact of strategic similarity in horizontal mergers: evidence from U.S. banking industry. The Academy of Management Journal 40(3):697-715.

Ranft, A.L., und M.D. Lord. 2002. Acquiring new technologies and capabilities: a grounded model of acquisition implementation. Organization Science 13(4):420-441.

Raveendran, M. 2020. Seeds of change: how current structure shapes the type and timing of reorganizations. Strategic Management Journal 41(1):27-54. 
Roberts, J. 2004. The modern firm: organizational design for performance and growth. New York: Oxfrod University Press.

Rouziès, A., H. Colman, und D. Angwin. 2019. Recasting the dynamics of post-acquisition integration: an embeddedness perspective. Long Range Planning 52(2):271-282.

Ruh, R.A., J.K. White, und R.R. Wood. 1975. Job involvement, values, personal background, participation in decision making, and job attitudes. The Academy of Management Journal 18(2):300-312.

Savović, S. 2017. Organizational culture differences and post-acquisition performance: The mediating role of employee attitudes. Leadership \& Organization Development Journal 38(5):719-741. https://doi. org/10.1108/LODJ-02-2016-0043.

Schein, E.H. 1985. Organizational culture and leadership: A dynamic view. San Francisco: Jossey-Bass.

Schuler, R.S. 1977. Role perceptions, satisfaction and performance moderated by organization level and participation in decision making. The Academy of Management Journal 20(1):159-165.

Schweiger, D.M., und E.N. Csiszar. 2002. Implementing international mergers and acquisitions. Human Resource Planning 16(1):53-70.

Schweiger, D.M., und Y. Weber. 1989. Strategies for managing human resources during mergers and acquisitions: an empirical investigation. Human Resource Planning 12(2):69-86.

Schweiger, D.M., J.M. Ivancevich, und F.R. Power. 1987. Executive actions for managing human resources before and after acquisition. The Academy of Management Executive 1(2):127-138.

Schweiger, D.M., und J.M. Ivancevich. 1985. Human resources: the forgotten factor in mergers and acquisitions. Personnel Administrator (30)11:47-61.

Schweizer, L. 2005. Organizational integration of acquired biotechnology companies into pharmaceutical companies: the need for a hybrid approach. The Academy of Management Journal 48(6):1051-1074.

Shelton, L.M. 1988. Strategic business fits and corporate acquisition: empirical evidence. Strategic Management Journal 9(3):279-287.

Shrivastava, P. 1986. Postmerger integration. Journal of Business Strategy 5(3):103-111.

Singh, H., und C.A. Montgomery. 1987. Corporate acquisition strategies and economic performance. Strategic Management Journal 8(4):377-386.

Sørensen, J.B. 2002. The strength of corporate culture and the reliability of firm performance. Administrative Science Quarterly 47(1):70-91.

Stahl, G.K., und A. Voigt. 2008. Do cultural differences matter in mergers and acquisitions? A tentative model and examination. Organization Science 19(1):160-176.

Teerikangas, S., und I. Thanos. 2018. Looking into the 'black box'-unlocking the effect of integration on acquisition performance. European Management Journal 36(3):366-380.

Trichterborn, A., D. Zu Knyphausen-Aufseß, und L. Schweizer. 2016. How to improve acquisition performance: the role of a dedicated M\&A function, M\&A learning process, and M\&A capability. Strategic Management Journal 37(4):763-773.

Uhlenbruck, K., M.A. Hitt, und M. Semadeni. 2006. Market value effects of acquisitions involving internet firms: a resource-based analysis. Strategic Management Journal 27(10):899-913.

Venkatraman, N. 1989. The concept of fit in strategy research: toward verbal and statistical correspondence. The Academy of Management Review 14(3):423-444.

Venkatraman, N., und J.C. Camillus. 1984. Exploring the concept of "Fit" in strategic management. The Academy of Management Review 9(3):513-525.

Vroom, V.H. 1959. Some personality determinants of the effects of participation. The Journal of Abnormal and Social Psychology 59(3):322-327.

Vroom, V.H., und P.W. Yetton. 1973. Leadership and decision-making. Pittsburgh: University of Pittsburgh Press.

Weber, Y. 2019. The role of organizational identity in post-merger integration. In The synergy of business theory and practice, 91-107. London: Palgrave Macmillan.

Weber, Y., und D.M. Schweiger. 1992. Top management culture conflict in mergers and acquisitions: a lesson from anthropology. The International Journal of Conflict Management, 3(4):285-302.

Wiersema, M.F., und H.P. Bowen. 2009. The use of limited dependent variable techniques in strategy research: issues and methods. Strategic Management Journal 30(6):679-692.

Yin, X., und E.J. Zajac. 2004. The strategy/governance structure fit relationship: theory and evidence in franchising arrangements. Strategic Management Journal 25(4):365-383.

Yli-Renko, H., E. Autio, und H.J. Sapienza. 2001. Social capital, knowledge acquisition, and knowledge exploitation in young technology-based firms. Strategic Management Journal 22(6-7):587-613.

Zaheer, A., X. Castaner, und D. Souder. 2013. Synergy sources, target autonomy, and integration in acquisitions. Journal of Management 39(3):604-632. 